\title{
Halogen Bonding Promotes Higher Dye-Sensitized Solar Cell Photovoltages
}

\author{
Supplementary Information
}

Sarah J. C. Simon ${ }^{1}$, Fraser G. L. Parlane ${ }^{1}$, Wesley B. Swords ${ }^{2}$, Cameron W. Kellett $^{1}$,

Chuan $\mathrm{Du}^{1}$, Brian Lam ${ }^{1}$, Rebecca K. Dean ${ }^{1}, \mathrm{Ke} \mathrm{Hu}^{2}$, Gerald J. Meyer*2 and Curtis P. Berlinguette*1 $^{* 1}$
${ }^{1}$ Departments of Chemistry and Chemical \& Biological Engineering, The University of British Columbia, 2036 Main Mall, Vancouver, BC V6T 1Z1, Canada
${ }^{2}$ Department of Chemistry, The University of North Carolina at Chapel Hill, Murray Hall 2202B, Chapel Hill, NC 27599-3290, USA.

\author{
Correspondance to \\ cberling@chem.ubc.ca \\ gjmeyer@email.unc.edu
}




\section{Contents}

1 Methods $\quad$ S4

1.1 Synthetic Methods . . . . . . . . . . . . . . . . . . . . S S4

1.2 Physical Methods . . . . . . . . . . . . . . . . . . . . . . S4

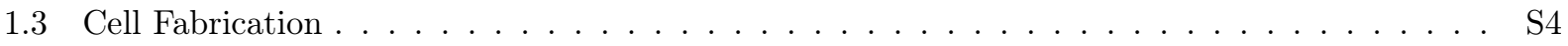

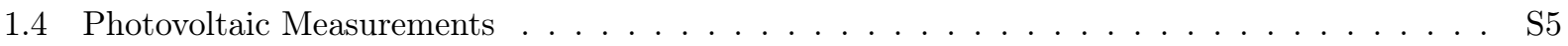

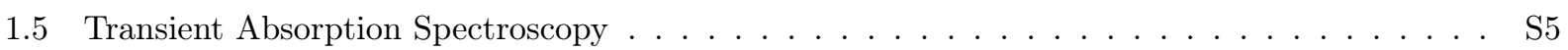

1.6 Computational Methods . . . . . . . . . . . . . . . . . . . . . . . . . . S6

$\begin{array}{lll}2 & \text { Results } & \text { S7 }\end{array}$

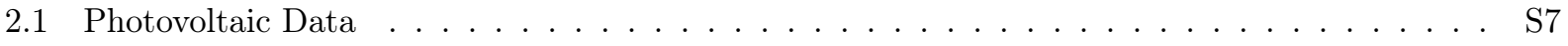

2.2 Transient Absorption Data $\ldots \ldots \ldots \ldots \ldots \ldots \ldots \ldots \ldots$

2.2 .1 Regeneration Kinetics . . . . . . . . . . . . . . . . . . . . . . . . . . S9

2.2.2 Charge Recombination Kinetics . . . . . . . . . . . . . . . . . . . . . . . . S10

2.2.3 Possible Intermolecular Electron Transfers . . . . . . . . . . . . . . . . . . . . . . S10

2.3 Computational Models and Natural Bond Orbital (NBO) Analysis . . . . . . . . . . . . . S11

2.3.1 Halogen-Iodide Interaction: Summary of NBO Analysis . . . . . . . . . . . . . . S11

2.3.2 Phenyl-Iodide Interaction: Summary of Computational Analysis . . . . . . . . . . S13

2.3.3 Amine-Iodide Interaction: Summary of Computational Analysis . . . . . . . . . . . . S15

2.3 .4 Optimized Geometries . . . . . . . . . . . . . . . . . . S16

3 References $\quad$ S24 


\section{List of Figures}

S1 Photocurrent Density-Voltage Curves . . . . . . . . . . . . . . . . . . . . S7

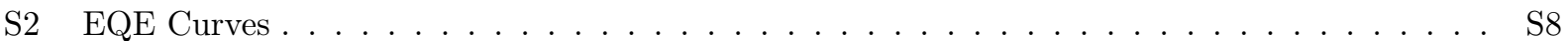

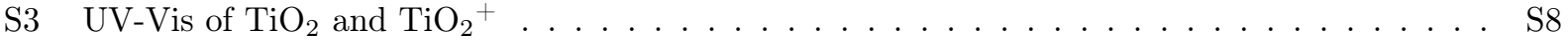

S4 Fluorescence Spectra of Dye-X Series . . . . . . . . . . . . . . . . . . . S8

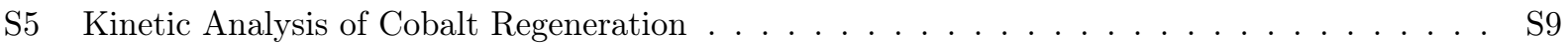

S6 Regeneration Rate Constants of Iodide and Cobalt Electrolytes . . . . . . . . . . . . . . S9

S7 Charge Recombination with Electrolyte Kinetics _ . . . . . . . . . . . . . . . . . . . S10

S8 Halogen-Iodide Interaction: DFT Interaction Structure . . . . . . . . . . . . . . . . S11

S9 Phenyl-Iodide Interaction: DFT Interaction Structure $\ldots \ldots \ldots \ldots$. . . . . . . . . S13

S10 Amine-Iodide Interaction: DFT Interaction Structure . . . . . . . . . . . . . . S15

S11 Electrostatic Potential Plot and Natural Population Analysis of Oxidized Dyes . . . . . . . S15

\section{List of Tables}

S1 Recombination Kinetics to Cobalt . . . . . . . . . . . . . . . . . . S10

S2 Calculated Halogen-Iodide Interactions . . . . . . . . . . . . . . . . . . S11

S3 Halogen-Iodide Interaction: Summary of NBO Analysis . . . . . . . . . . . . . . . . S12

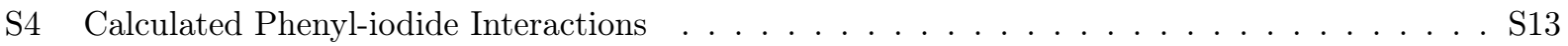

S5 Phenyl-Iodide Interaction: Summary of NBO Analysis . . . . . . . . . . . . . . . . . . . S14

S6 Calculated Amine-Iodide Interactions . . . . . . . . . . . . . . . . . . . . . . S15 


\section{Methods}

\section{$1.1 \quad$ Synthetic Methods}

The synthetic methods for synthesizing and characterizing the Dye-X series[1] and 1,3-dimethylimidazolium iodide (DMII)[2] were prepared and characterized as previously described.

\subsection{Physical Methods}

Acetonitrile $\left(\mathrm{CH}_{3} \mathrm{CN}\right)$ was purchased from Honeywell (Burdick and Jackson, 99.9\%) and used as received. Anhydrous sodium iodide (99.999\%), tetrabutylammonium tetrafluoroborate ( $>98.0 \%)$, and sodium perchlorate $(>98 \%)$ were purchased from Sigma Aldrich and used as received. Electrochemical data of the dyes in solution were recorded with a CHI660D potentiostat using a platinum wire counter electrode, platinum wire reference electrode, and a platinum working electrode. A $0.1 \mathrm{M} \mathrm{TBABF}_{4}$ electrolyte solution in acetonitrile at ambient temperature was used for all dyes. Electrochemical data of the dyes on sensitized $\mathrm{TiO}_{2}$ thin film on an FTO substrate as the working electrode were recorded with a BASi CV-50W potentiostat. A platinum mesh counter electrode and $\mathrm{BASi}$ aqueous $\mathrm{Ag} / \mathrm{AgCl}$ reference electrode in a $0.3 \mathrm{M} \mathrm{NaClO}_{4}$ supporting electrolyte were used for the dyes adsorbed to $\mathrm{TiO}_{2}$. All electrochemical and spectroelectrochemical measurements were externally referenced to ferrocene $\left(\mathrm{Fc} / \mathrm{Fc}^{+},+640 \mathrm{mV}\right.$ vs. NHE). CVs were acquired at a scan rate of $100 \mathrm{mV} \mathrm{s}^{-1}$. Fluorescence measurements were performed on an Agilent Cary Eclipse spectrophotometer equipped with a xenon flash lamp. UV-vis absorption spectra of sensitized $\mathrm{TiO}_{2}$ thin films were recorded using a Varian Cary $60 \mathrm{UV}$-vis spectrophotometer with a resolution of $1 \mathrm{~nm}$.

\subsection{Cell Fabrication}

Photoanodes were prepared by screen printing $\mathrm{TiO}_{2}$ pastes (2 layers of 18-NRT and 1 layer of WER4-0, obtained from Dyesol) on on fluorine-doped tin-oxide [FTO; TEC7 $\left.\left(7 \Omega \mathrm{cm}^{-2}\right)\right]$ coated glass. The active area of the $\mathrm{TiO}_{2}$ electrodes was $0.13 \mathrm{~cm}^{2}$ with a $13-15 \mu$ m thickness. The $\mathrm{TiO}_{2}$ substrates (anodes) were treated with $\mathrm{TiCl}_{4}(\mathrm{aq})(0.05 \mathrm{M})$ at $70{ }^{\circ} \mathrm{C}$ for $30 \mathrm{~min}$ followed by a rinse with $\mathrm{H}_{2} \mathrm{O}$. Anodes were then heated to $500{ }^{\circ} \mathrm{C}$ for $35 \mathrm{~min}$ in an ambient atmosphere and left to cool to $80{ }^{\circ} \mathrm{C}$ prior to immersing into an acetonitrile solution containing the dye $(0.25 \mathrm{mM})$ for $20 \mathrm{~h}$. The stained anodes were then rinsed with copious amounts of $\mathrm{CH}_{3} \mathrm{CN}$ and dried. FTO-coated glass substrates for fabricating the cathodes were prewashed with $0.1 \mathrm{M} \mathrm{HCl} / \mathrm{EtOH}$ solution, sonicated in acetone for $10 \mathrm{~min}$, rinsed with EtOH, and dried prior to heating to $400{ }^{\circ} \mathrm{C}$ for $30 \mathrm{~min}$ in ambient atmosphere and and allowing to cool to room temperature. A drop of $2 \mathrm{mg} \mathrm{mL}-1 \mathrm{H}_{2} \mathrm{PtCl}_{6} \cdot 6 \mathrm{H}_{2} \mathrm{O}$ in EtOH was placed on the cathodes and heated to $400{ }^{\circ} \mathrm{C}$ for 30 min in an ambient atmosphere and left to cool to room temperature. Cell assembly was performed in an isolated chamber purged with dry atmosphere. The anodes were rinsed with acetonitrile and dried prior to sealing with the cathode in a sandwich configuration using a thermoplastic gasket (pre-cut $30 \mu \mathrm{m}$ Dupont 
Surlyn $^{\mathrm{TM}}$ films, obtained from Dysol) via a mechanical hot press. Iodide electrolyte used for this study was made with $1.0 \mathrm{M}$ 1,3-dimethylimidazolium iodide (DMII), $60 \mathrm{mM} \mathrm{I}_{2}, 0.5 \mathrm{M}$ tert-butylpyridine, $0.05 \mathrm{M} \mathrm{NaI}$, and $0.1 \mathrm{M}$ guanidinium thiocyanate in a mixed solvent system of acetonitrile and valeronitrile $(85: 15, \mathrm{v} / \mathrm{v})$. Cobalt electrolyte used for this study was made with $0.02 \mathrm{M} \mathrm{Co}(\mathrm{bpy})_{3}\left(\mathrm{PF}_{6}\right)_{3}, 0.2 \mathrm{M} \mathrm{Co}(\mathrm{bpy})_{3}\left(\mathrm{PF}_{6}\right)_{2}, 0.1$ $\mathrm{M} \mathrm{LiClO}_{4}, 0.2 \mathrm{M}$ tert-butylpyridine in acetonitrile. The electrolyte was introduced into the two-sandwiched electrodes via micropipette through holes pre-drilled in the cathode. The holes were sealed with Bynel and

a glass microscope slide. After sealing, silver paint was brushed on one end of the anode and cathode on the conductive side for all cells.

\subsection{Photovoltaic Measurements}

Photovoltaic measurements were recorded with a TriSOL solar simulator (SS1012) equipped with a class A $300 \mathrm{~W}$ xenon light source powered by a Newport power supply (model 69911). The light output (area = $52 \mathrm{~mm} \times 52 \mathrm{~mm}$ ) was calibrated to AM 1.5 using a Newport Oriel correction filter to reduce the spectral mismatch in the region of 350-700 nm to less than 1.5\%. The power output of the lamp was measured to 1 Sun $\left(100 \mathrm{~mW} \mathrm{~cm}^{-2}\right)$ using a certified Newport Oriel Si reference cell (Model SN 679). The current-voltage (I-V) characteristic of each cell was obtained by applying an external potential bias to the cell and measuring the generated photocurrent with a Keithley digital source meter (model 2400). All cells were placed and fixed with cellophane tape on a lab jack covered with aluminum foil, before attaching to the Keithley digital source meter. IPCE measurements were performed on a Newport Oriel IQE $200^{\mathrm{TM}}$. All measurements were made in $\mathrm{AC}$ mode at a $10 \mathrm{~Hz}$ chopping frequency under a bias light.

\subsection{Transient Absorption Spectroscopy}

Nanosecond transient absorption measurements of iodide regeneration rates were obtained with an apparatus similar to that which has been previously described.[3][4] Samples for Transient Absorption were prepared as described previously.[3] Briefly, samples were excited by a frequency doubled Q-switched, pulsed Nd:YAG laser (Quantel USA (BigSky) Brilliant B; $532 \mathrm{~nm}, 5-6 \mathrm{~ns}$ full width at half-maximum (fwhm), $1 \mathrm{~Hz}, \sim 10$ $\mathrm{mm}$ in diameter) directed $45^{\circ}$ to the film surface. A Glan laser polarizer was employed in the laser path to attenuate the pulse fluence. A $150 \mathrm{~W}$ xenon arc lamp, pulsed with $70 \mathrm{~V}$, served as the probe beam (Applied Photophysics) and was aligned orthogonally to the excitation laser. Detection was achieved with a monochromator (Spex 1702/04) optically coupled to an R928 photomultiplier tube (Hamamatsu). Transient data were acquired on a computer-interfaced digital oscilloscope (LeCroy 9450, Dual $350 \mathrm{MHz}$ ) with 2.5 ns resolution terminated at $50 \Omega$. For full spectral generation, laser pulses were averaged 30 times at each collected wavelength over the range $370-800 \mathrm{~nm}$. Single wavelength measurement had to average at least 150 laser pulses to achieve satisfactory S/N. Kinetic data fitting and spectral modeling was performed in Origin 9.1, and least-squares error minimization was accomplished using the Levenberg-Marquardt iteration method. Transient absorbance spectra of cobalt regeneration was collected using a Princeton Instruments 
Spectra Pro 2300i Imaging Triple Grating Monochromator/Spectrograph with a Hamamatsu Dynamic Range Streak Camera (excitation source: EKSPLA PL2241 Nd:YAG laser, $\lambda=532 \mathrm{~nm}$, fwhm=35 ps).

\subsection{Computational Methods}

Quantum mechanical calculations were run using the Gaussian 09 computational package.[5] All calculations were carried out using the unrestricted M06-2X functional with a conductor-like polarizable continuum solvation model (CPCM) of acetonitrile, except where noted.[6-9] All geometries were optimized to a minimum and frequency calculations performed at the same level of theory to verify the absence of imaginary frequencies. The initial dye structures were optimized as previously described.[1] $\beta$-LUSOs were visualized using the Jmol version 13.0 software package from the formatted checkpoint files at an iso-value of $0.05,[10]$ and the electrostatic potential was plotted using the GaussView 5.0.8 software package over the total electron density at an iso-value of 0.001.[11] The dye-iodide interaction geometries were optimized using an ultrafine integration grid (99 radial shells, 590 angular points) and the aug-cc-pVDZ-PP basis set on bromine and iodine, aug-cc-pVDZ on fluorine and chlorine, and cc-pVDZ on all other atoms, while subsequent calculations were performed using an ultrafine integration grid and aug-cc-pVTZ-PP on bromine and iodine, aug-cc-pVTZ on fluorine and chlorine, and cc-pVTZ on all other atoms.[12-18] Parameters for the aug-cc-pVDZ-PP and aug-cc-pVTZ-PP basis sets were obtained from the ESML basis set exchange.[19, 20] To ensure that charges were distributed appropriately on the interacting pairs, a fragment guess was first generated specifying a negative charge on iodide and all subsequent calculations were performed reading their initial guess from a checkpoint file based off this fragment guess. The basis set superposition error (BSSE) for the dye-iodide interactions was estimated in the gas phase using the counterpoise method for the interacting dimers.[21-24] Since BSSE is dependent upon the degree of basis set overlap between two monomers, the contributions of this error to the energy of the noninteracting systems is expected to be negligible. The interaction energy $\left(\Delta E_{\text {int }}\right)$ was estimated by subtracting the single point energy of the non-interacting pairs $\left(E_{12 \AA}\right.$; modeled as described below) from the single point energy of the interacting complexes $\left(E_{\text {complex }}\right)$ :

$$
\Delta E_{\text {int }}=E_{\text {complex }}-E_{12 \AA}
$$

The calculated single point energies were corrected for BSSE as described above. The geometry of the non-interacting pairs were not optimized, but rather generated from the initial dye geometries by placing an

iodine atom $12 \AA$ away from the dye, in line with the carbon-halogen bond axis. Second-order perturbation analysis of intermolecular interactions was performed with the NBO 6.0 program.[25-34] 


\section{Results}

\subsection{Photovoltaic Data}

A.)

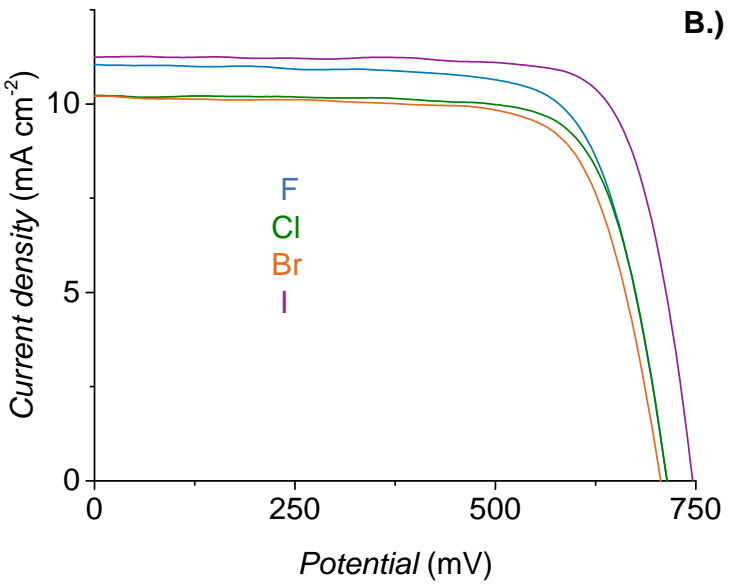

B.)

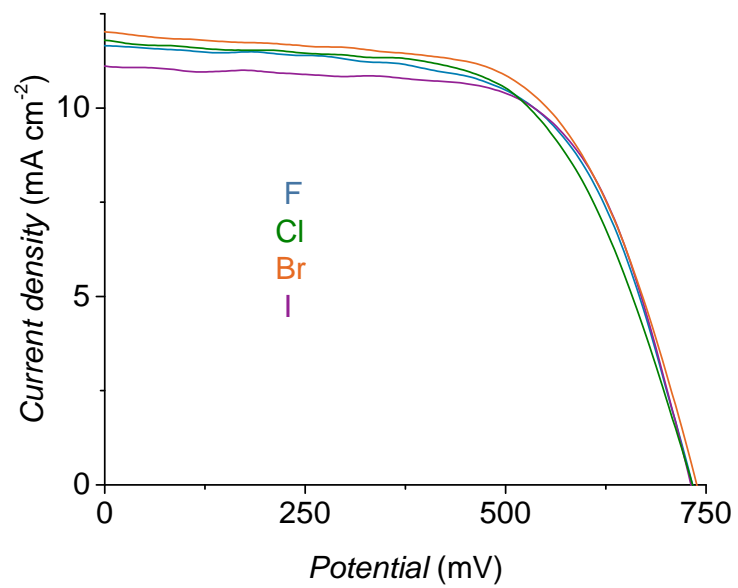

Figure S1: Representative photocurrent density-voltage curves of Dye-X cells measured under irradiation of one sun with an electrolyte composed of (A) an iodide and (B) cobalt electrolyte. 


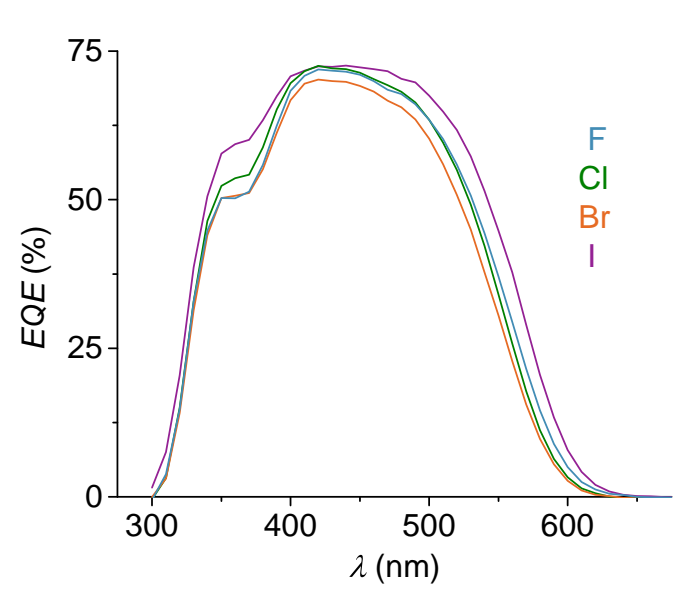

Figure S2: Incident-photon-to-current efficiency (IPCE) for the Dye-X series with $\mathrm{I}_{3}^{-} / \mathrm{I}^{-}$electrolyte

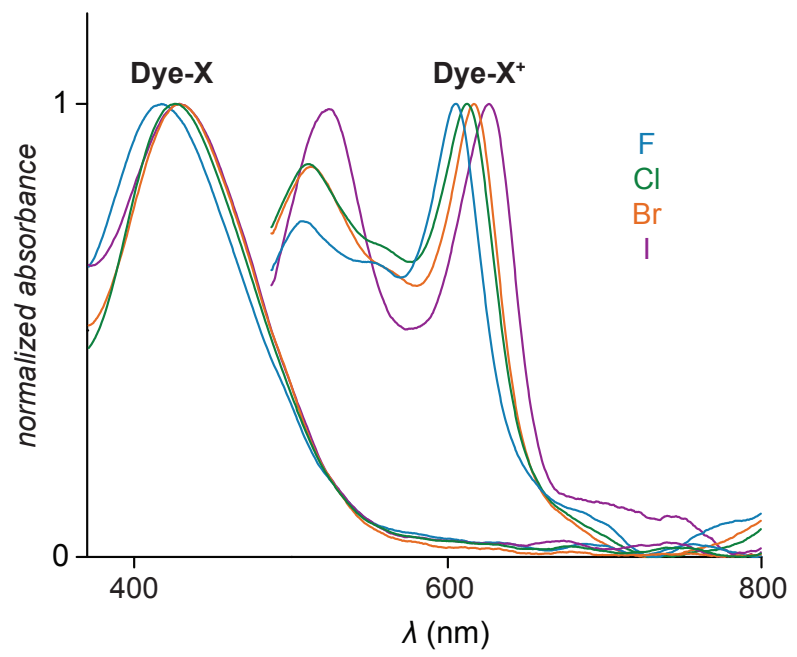

Figure S3: Normalized UV-vis absorption spectra for Dye-X and Dye- $\mathbf{X}^{+}$immobilized on $\mathrm{TiO}_{2}$.

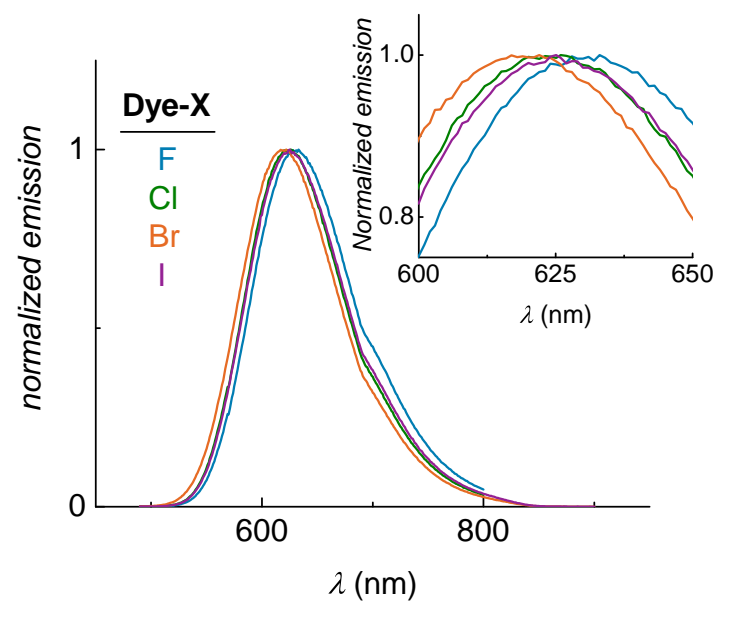

Figure S4: Normalized fluorescence spectra for Dye-X in DCM. 


\subsection{Transient Absorption Data}

\subsubsection{Regeneration Kinetics}

A.)

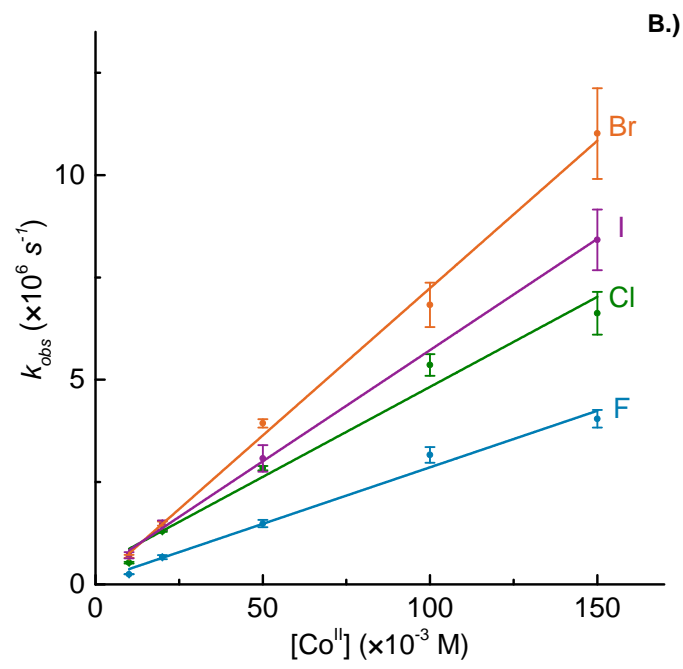

B.)
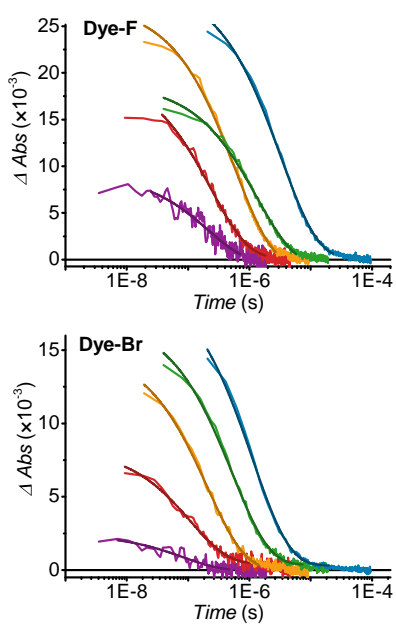
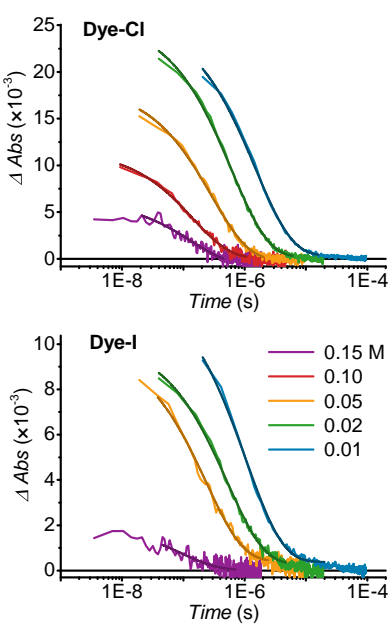

Figure S5: A) Overlaid plots of obtained kobs from kinetic fitting of the single wavelength kinetic data. B) Regeneration kinetic data of $\mathrm{TiO}_{2}\left(\mathrm{e}^{-}\right) /$Dye- $\mathrm{X}^{+}$and $\mathrm{Co}^{\mathrm{II}}$ at cobalt concentrations of $10-150 \mathrm{mM}$ in $\mathrm{NaClO}_{4}$ $\left(0.3 \mathrm{M}\right.$ in $\left.\mathrm{CH}_{3} \mathrm{CN}\right)$ solutions. Dyes were excited at $532 \mathrm{~nm}$ and kinetic data was generated by averaging traces $10 \mathrm{~nm}$ to the peak of the oxidized spectrum.

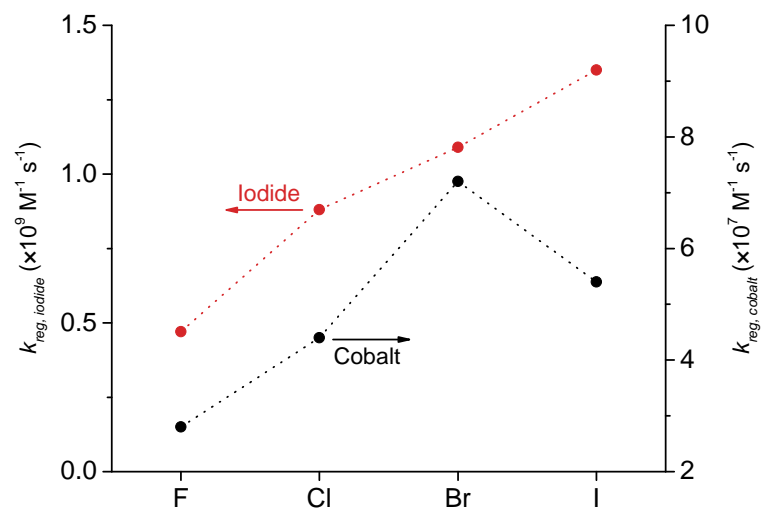

Figure S6: Regeneration rate constants of $\mathrm{TiO}_{2}\left(\mathrm{e}^{-}\right) / \mathbf{D y e}-\mathrm{X}^{+}$by I- (red) and $\mathrm{Co}^{\mathrm{II}}$ (black). Regeneration rate constants for iodide closely follow the increasing degree of halogen bonding, whereas regeneration via cobalt more closely follows the driving force. 


\subsubsection{Charge Recombination Kinetics}
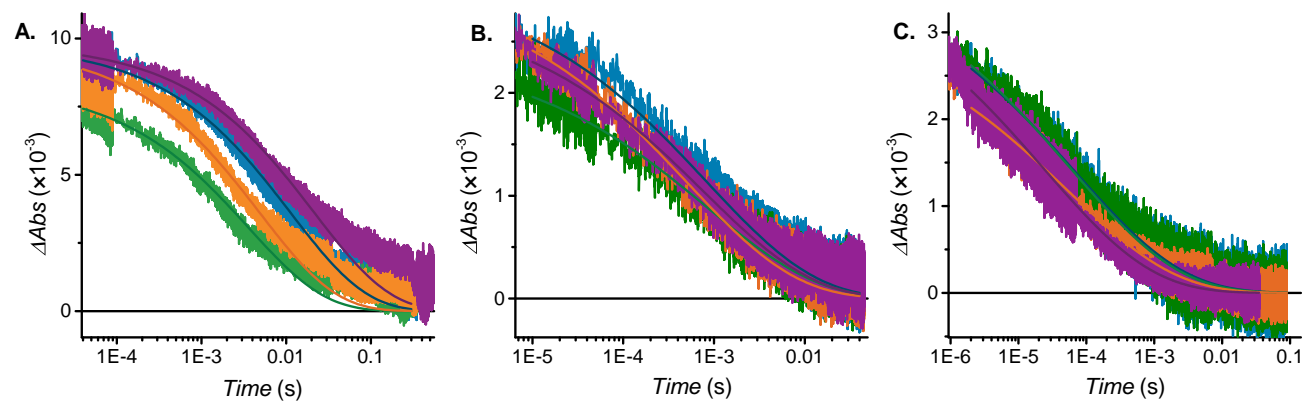

Figure S7: Recombination from $\mathrm{TiO}_{2}$ electrons to a) $\mathrm{I}_{3}{ }^{-}$at half surface coverage, b) $\mathrm{I}_{3}{ }^{-}$at full surface coverage, and c) $\mathrm{Co}^{\mathrm{III}}$ at full surface coverage. The electrolyte was either a,b) $0.3 \mathrm{M} \mathrm{NaI}$ or c) $0.2 \mathrm{M} \mathrm{Co}^{\mathrm{II}}(\mathrm{bpy})_{3}$ in $0.3 \mathrm{NaClO}_{4}$ in $\mathrm{CH}_{3} \mathrm{CN}$. Fits are to the KWW function with the stretching parameter $\beta$ fixed at a) 0.70 , b) 0.32 , or c) 0.28 . Recombination rates are reported in Table $\mathrm{S} 1$.

Table S1: Recombination kinetics

\begin{tabular}{|c|c|c|c|c|c|c|}
\hline \multirow{3}{*}{$\begin{array}{r}\text { Electrolyte: } \\
\text { Coverage: }\end{array}$} & \multicolumn{4}{|c|}{$\begin{array}{l}\text { Iodide }^{[\mathrm{a}]} \\
\left(0.6 \mathrm{M} \mathrm{I}^{-}\right)\end{array}$} & \multirow{2}{*}{\multicolumn{2}{|c|}{$\begin{array}{c}\text { Cobalt }^{[\mathrm{b}]} \\
\left(0.22 \mathrm{M} \mathrm{Co}{ }^{\mathrm{II}}\right) \\
100 \%\end{array}$}} \\
\hline & \multicolumn{2}{|c|}{$50 \%$} & \multicolumn{2}{|c|}{$100 \%$} & & \\
\hline & $\begin{array}{l}k_{r e c} \\
\left(s^{-1}\right)\end{array}$ & $\begin{array}{c}\tau_{r e c} \\
\left(\times 10^{-3} s\right)\end{array}$ & $\begin{array}{l}k_{r e c} \\
\left(s^{-1}\right)\end{array}$ & $\begin{array}{c}\tau_{r e c} \\
\left(\times 10^{-3} s\right)\end{array}$ & $\begin{array}{c}k_{r e c} \\
\left(s^{-1}\right)\end{array}$ & $\begin{array}{c}\tau_{r e c} \\
\left(\times 10^{-4} s\right)\end{array}$ \\
\hline Dye-F $^{+}$ & $55 \pm 20$ & $18 \pm 15$ & $210 \pm 80$ & $5 \pm 4$ & $900 \pm 100$ & $11 \pm 3$ \\
\hline Dye-Cl ${ }^{+}$ & $220 \pm 85$ & $5 \pm 4$ & $230 \pm 60$ & $4 \pm 2$ & $1100 \pm 300$ & $9 \pm 5$ \\
\hline Dye-Br ${ }^{+}$ & $120 \pm 32$ & $8 \pm 5$ & $280 \pm 40$ & $4 \pm 1$ & $1300 \pm 700$ & $8 \pm 12$ \\
\hline Dye-I ${ }^{+}$ & $40 \pm 15$ & $25 \pm 22$ & $210 \pm 50$ & $5 \pm 2$ & $2900 \pm 200$ & $3.4 \pm 0.5$ \\
\hline
\end{tabular}

Recombination from $\mathrm{TiO}_{2}$ electrons to a) $\mathrm{I}_{3}{ }^{-}$and b) $\mathrm{Co}^{\mathrm{III}}$ at either half or full surface coverage.

The electrolyte was either a) $0.3 \mathrm{M} \mathrm{NaI}$ or b) $0.2 \mathrm{M} \mathrm{Co}^{\mathrm{II}}(\mathrm{bpy})_{3}$ in $0.3 \mathrm{NaClO}_{4}$ in $\mathrm{CH}_{3} \mathrm{CN}$.

\subsubsection{Possible Intermolecular Electron Transfers}

\section{Regeneration}

$\mathrm{TiO}_{2}\left(\mathrm{e}^{-}\right) / \mathrm{Dye}^{+}+2 \mathrm{I}^{-} \rightarrow \mathrm{TiO}_{2}\left(\mathrm{e}^{-}\right) / \mathrm{Dye}+\mathrm{I}_{2}{ }^{\cdot-}$

$\mathrm{TiO}_{2}\left(\mathrm{e}^{-}\right) / \mathrm{Dye}^{+}+\mathrm{Co}^{\mathrm{II}} \rightarrow \mathrm{TiO}_{2}\left(\mathrm{e}^{-}\right) /$Dye $+\mathrm{Co}^{\mathrm{III}}$

Back Electron Transfer (BET)

$\mathrm{TiO}_{2}\left(\mathrm{e}^{-}\right) / \mathrm{Dye}^{+} \rightarrow \mathrm{TiO}_{2} /$ Dye

Charge Recombination with Electrolyte (CR)

$\mathrm{TiO}_{2}\left(\mathrm{e}^{-}\right) / \mathrm{Dye}^{+}+\mathrm{I}_{3}^{-} \rightarrow \mathrm{TiO}_{2} / \mathrm{Dye}^{+}+\mathrm{I}_{2}{ }^{--}+\mathrm{I}^{-}$

$\mathrm{TiO}_{2}\left(\mathrm{e}^{-}\right) / \mathrm{Dye}^{+}+\mathrm{Co}^{\mathrm{III}} \rightarrow \mathrm{TiO}_{2} / \mathrm{Dye}^{+}+\mathrm{Co}^{\mathrm{II}}$ 


\subsection{Computational Models and Natural Bond Orbital (NBO) Analysis}

\subsubsection{Halogen-Iodide Interaction: Summary of NBO Analysis}

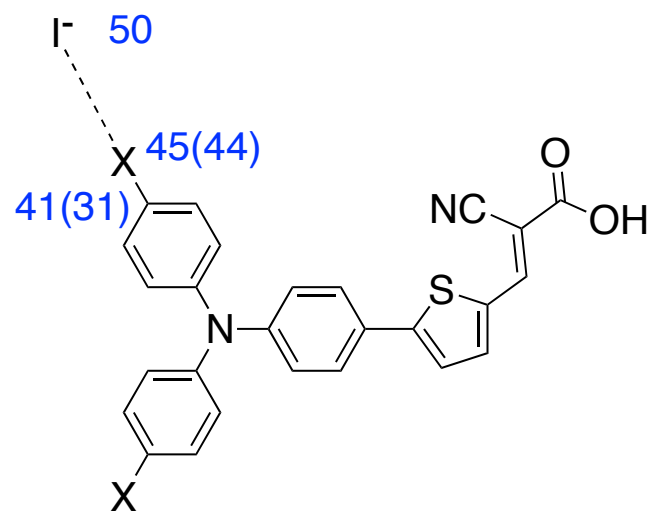

Figure S8: Schematic representation of Dye- $\mathbf{X}^{+} \ldots \mathrm{I}^{-}$halogen-iodide interaction geometries. Selected atom numbers used in NBO analysis are listed in blue; numbers in parentheses apply to $\mathbf{D y e - B r}{ }^{+}$only.

Table S2: Calculated Halogen-Iodide Distances, Interaction Energies, and Stabilization Energies Describing the Interaction of $\mathbf{D y e}-\mathbf{X}^{+}$with Iodide via the halogen substituent, as shown in Figure S8.

\begin{tabular}{llll}
\hline Dye & $\begin{array}{l}\mathrm{d} \times \cdots \mathrm{I} \\
(\AA)^{a}\end{array}$ & $\begin{array}{l}\text { van der Waals distance } \\
(\AA)^{b}\end{array}$ & $\begin{array}{l}\Delta E_{\text {int }} \\
\left.(\mathrm{kcal} \mathrm{mol})^{-1}\right)^{c}\end{array}$ \\
\hline Dye-F $^{+}$ & 4.33 & 3.53 & -0.58 \\
Dye-Cl $^{+}$ & 3.77 & 3.81 & -1.20 \\
Dye-Br $^{+}$ & 3.66 & 3.91 & -2.28 \\
Dye-I $^{+}$ & 3.68 & 4.04 & -3.63 \\
\hline
\end{tabular}

${ }^{a}$ Optimized internuclear distance between the halogen atom and $\mathrm{I}^{-}$.

${ }^{b}$ Internuclear distance between the halogen atom and $\mathrm{I}^{-}$predicted by van der Waals radii. ${ }^{c}$ Interaction energy corrected for basis set superposition error. 
Table S3: Key second order iodide / dye interactions based on optimized Dye- $\mathbf{X}^{+} \ldots \mathrm{I}^{-}$ halogen-iodide interaction structures (Figure S8). Stabilization energy $\left(E^{2}\right)$ values and $F_{i j}$ values are reported as the sum of alpha and beta terms for equivalent interactions in kcal mol ${ }^{-1}$ and a.u., respectively; $\epsilon_{j}-\epsilon_{i}$ values are reported as the average of alpha and beta terms in a.u. Only interactions with $E^{2}$ greater than $1.0 \mathrm{kcal} \mathrm{mol}^{-1}$ are included.

\begin{tabular}{|c|c|c|c|c|c|}
\hline Dye & Donor & Acceptor & $\begin{array}{l}E^{2} \\
k c a l m o l^{-1}\end{array}$ & $\begin{array}{l}F_{i j} \\
(a . u .)\end{array}$ & $\begin{array}{l}\epsilon_{j}-\epsilon_{i} \\
(\text { a.u. })\end{array}$ \\
\hline Dye-F ${ }^{+}$ & $\begin{array}{l}65 \alpha . \mathrm{LP}(4) \mathrm{I} 50 \\
63 \beta . \mathrm{LP}(4) \mathrm{I} 50\end{array}$ & $\begin{array}{l}\text { 198a. BD*(1) C41 - F45 } \\
198 \beta . \mathrm{BD}^{*}(1) \mathrm{C} 41-\mathrm{F} 45\end{array}$ & $0.06^{\mathrm{a}}$ & 0.012 & 0.61 \\
\hline Dye-Cl $^{+}$ & $\begin{array}{l}75 \alpha . \text { LP (4) I50 } \\
71 \beta . \text { LP (4) I50 }\end{array}$ & $\begin{array}{l}206 \alpha . \mathrm{BD}^{*}(1) \mathrm{C} 41-\mathrm{Cl} 45 \\
206 \beta . \mathrm{BD}^{*}(1) \mathrm{C} 41-\mathrm{Cl} 45\end{array}$ & 1.73 & 0.052 & 0.48 \\
\hline Dye-Br ${ }^{+}$ & $\begin{array}{l}82 \alpha . \mathrm{LP}(4) \text { I50 } \\
79 \beta . \mathrm{LP}(4) \text { I50 }\end{array}$ & $\begin{array}{l}201 \alpha . \mathrm{BD}^{*}(1) \mathrm{C} 31-\mathrm{Br} 45 \\
200 \beta . \mathrm{BD}^{*}(1) \mathrm{C} 31-\mathrm{Br} 45\end{array}$ & 3.98 & 0.074 & 0.43 \\
\hline Dye-I ${ }^{+}$ & $\begin{array}{l}82 \alpha . \text { LP (4) I50 } \\
79 \beta . \text { LP (4) I50 }\end{array}$ & $\begin{array}{l}214 \alpha . \mathrm{BD}^{*}(1) \mathrm{C} 41-\mathrm{I} 45 \\
214 \beta . \mathrm{BD}^{*}(1) \mathrm{C} 41-\mathrm{I} 45\end{array}$ & 6.13 & 0.088 & 0.40 \\
\hline
\end{tabular}

${ }^{a}$ No interactions were found above $1.0 \mathrm{kcal} \mathrm{mol}^{-1}$ for $\mathbf{D y e}-\mathbf{F}^{+} \ldots \mathrm{I}^{-}$, therefore the strongest calculated interaction is reported instead.

\section{Halogen-iodide interaction: Hybridization and Polarization data for selected NBOs}

*Only orbital coefficients greater than $1.00 \%$ were reported.

\section{Dye- $F^{+}$}

65 $\alpha$. LP(4) I50: 100\% I50 (99.13\% p)

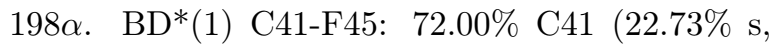

$77.09 \%$ p); $28.00 \%$ F45 (29.57\% s, $70.13 \%$ p)

63ß. LP(4) I50: 100\% I50 (99.13\% p)

198ß. BD*(1) C41-F45: $72.21 \%$ C41 $(22.77 \%$ s,

$77.05 \%$ p); $27.79 \%$ F45 (29.88\% s, $69.82 \%$ p)

\section{Dye- $\mathrm{Cl}^{+}$}

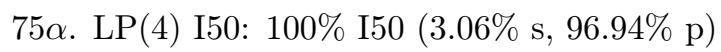

206a. BD*(1) C41-Cl45: 54.30\% C41 (23.54\% s,

$76.24 \%$ p); $45.70 \%$ Cl45 (17.76\% s, $81.47 \%$ p)

71ß. LP(4) I50: $100 \%$ I50 (3.08\% s, $96.92 \%$ p)

206\%. BD*(1) C41-Cl45: 54.68\% C41 (23.54\% s,

$76.24 \%$ p); $45.32 \%$ Cl45 (17.95\% s, $81.28 \%$

\section{Dye-Br ${ }^{+}$}

82 $\alpha$. LP(4) I50: $100 \%$ I50 (4.21\% s, $95.78 \%$ p)

201 $\alpha . \mathrm{BD}^{*}(1) \mathrm{C} 31-\mathrm{Br} 44: 49.88 \%$ C31 (22.93\% s, $76.81 \%$ p); $50.12 \%$ Br44 (12.70\% s, $86.78 \%$ p) 79ß. LP(4) I50: $100 \%$ I50 (5.33\% s, $94.67 \%$ p) 200 $\beta$. BD*(1) C31-Br44: 50.35\% C31 (22.91\% s, $76.82 \%$ p); $49.65 \%$; Br44 (12.89\% s, $86.61 \%$ p)

\section{Dye-I ${ }^{+}$}

82 $\alpha$. LP(4) I50: $100 \%$ I50 (4.97\% s, $95.03 \%$ p)

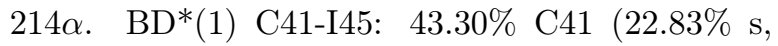
$76.89 \%$ p); $56.70 \%$ I45 (9.28\% s, $90.32 \%$ p)

79 . LP(4) I50: $100 \%$ I50 (5.01\% s, 94.99\% p)

214ß. BD*(1) C41-I45: $43.97 \%$ C41 $(22.78 \%$ s, $76.94 \%$ p); $56.03 \%$ I 45 (9.47\% s, $90.13 \%$ p) 


\subsubsection{Phenyl-Iodide Interaction: Summary of Computational Analysis}

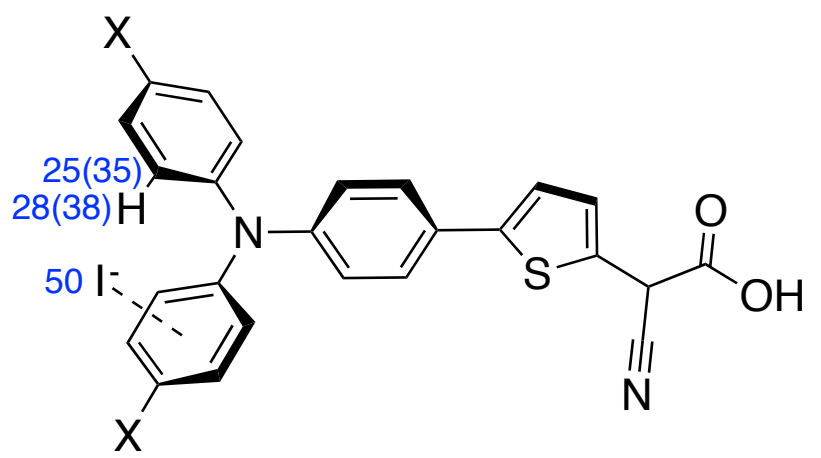

Figure S9: Schematic representation of Dye- $\mathbf{X}^{+} \ldots \mathrm{I}^{-}$iodide-phenyl interaction geometries (I-Ph). Selected atom numbers used in NBO analysis are listed in blue; numbers in parentheses apply to $\mathbf{D y e}-\mathbf{B r}^{+}$only.

Table S4: Calculated Halogen-Iodide Distances, Interaction Energies, and Stabilization Energies Describing the Interaction of $\mathbf{D y e}-\mathbf{X}^{+}$with Iodide in the I-Ph interaction geometries (Figure S9).

\begin{tabular}{lllll}
\hline Dye & $\begin{array}{l}\mathrm{d}_{\mathrm{Ph} \ldots \mathrm{I}} \ldots \mathrm{I} \\
(\AA)^{a}\end{array}$ & $\begin{array}{l}\mathrm{d}_{\mathrm{H} \ldots \mathrm{I}}(\AA)^{b} \\
(\AA)^{c}\end{array}$ & $\begin{array}{l}\text { van der Waals distance } \\
\left(\AA E_{\text {int }}\right. \\
\left(\mathrm{kcal} \mathrm{mol}^{-1}\right)^{d}\end{array}$ \\
\hline Dye-F $^{+}$ & 2.99 & 2.92 & 3.26 & -3.92 \\
Dye-Cl $^{+}$ & 4.03 & 2.93 & 3.26 & -4.03 \\
Dye-Br $^{+}$ & 3.56 & 2.94 & 3.26 & -3.94 \\
Dye- $^{+}$ & 2.87 & 2.94 & 3.26 & -3.97 \\
\hline
\end{tabular}

${ }^{a}$ Optimized internuclear distance between the iodide ion and the plane of the phenyl ring. ${ }^{b}$ Optimized internuclear distance between the iodide ion and the nearest $\mathrm{H}$ of the adjacent phenyl ring. ${ }^{c}$ Internuclear distance between the hydrogen atom and iodide ion predicted by van der Waals radii. ${ }^{d}$ Interaction energy corrected for basis set superposition error. 
Table S5: Key second order iodide / dye interactions based on optimized Dye- $\mathbf{X}^{+} \ldots \mathrm{I}^{-}$ $\mathrm{I}-\mathrm{Ph}$ interaction structures (Figure S9). Stabilization energy $\left(E^{2}\right)$ values and $F_{i j}$ values are reported as the sum of alpha and beta terms for equivalent interactions in $\mathrm{kcal} \mathrm{mol}^{-1}$ and a.u., respectively; $\epsilon_{j}-\epsilon_{i}$ values are reported as the average of alpha and beta terms in a.u. Only interactions with $E^{2}$ greater than $1.0 \mathrm{kcal} \mathrm{mol}^{-1}$ are included.

\begin{tabular}{|c|c|c|c|c|c|}
\hline Dye & Donor & Acceptor & $\begin{array}{l}E^{2} \\
k c a l m o l^{-1}\end{array}$ & $\begin{array}{l}F_{i j} \\
(a . u .)\end{array}$ & $\begin{array}{l}\epsilon_{j}-\epsilon_{i} \\
(\text { a.u. })\end{array}$ \\
\hline Dye-F $^{+}$ & $\begin{array}{l}65 \alpha . \operatorname{LP}(4) \text { I50 } \\
64 \beta . \operatorname{LP}(4) \text { I50 }\end{array}$ & $\begin{array}{l}175 \alpha . \mathrm{BD}^{*}(1) \mathrm{C} 25-\mathrm{H} 28 \\
176 \beta . \mathrm{BD}^{*}(1) \mathrm{C} 25-\mathrm{H} 28\end{array}$ & 3.66 & 0.095 & 0.78 \\
\hline Dye-Cl ${ }^{+}$ & $\begin{array}{l}73 \alpha . \text { LP (4) I50 } \\
71 \beta . \text { LP (4) I50 }\end{array}$ & 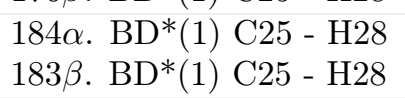 & 3.53 & 0.093 & 0.78 \\
\hline Dye-Br ${ }^{+}$ & $\begin{array}{l}81 \alpha . \text { LP (4) I50 } \\
79 \beta . \text { LP (4) I50 }\end{array}$ & $\begin{array}{l}206 \alpha . \mathrm{BD}^{*}(1) \mathrm{C} 35-\mathrm{H} 38 \\
205 \beta . \mathrm{BD}^{*}(1) \text { C35 - H38 }\end{array}$ & 3.46 & 0.092 & 0.77 \\
\hline Dye-I $^{+}$ & $\begin{array}{l}81 \alpha . \mathrm{LP}(4) \text { I50 } \\
79 \beta . \mathrm{LP}(4) \text { I50 }\end{array}$ & $\begin{array}{l}192 \alpha . \mathrm{BD}^{*}(1) \mathrm{C} 25-\mathrm{H} 28 \\
191 \beta . \mathrm{BD}^{*}(1) \mathrm{C} 25-\mathrm{H} 28\end{array}$ & 3.39 & 0.092 & 0.77 \\
\hline
\end{tabular}

Phenyl-iodide interaction: Hybridization and Polarization data for selected NBOs

*Only orbital coefficients greater than $1.00 \%$ were reported.

\section{Dye-F ${ }^{+}$}

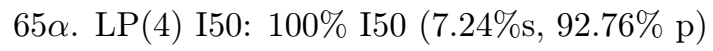

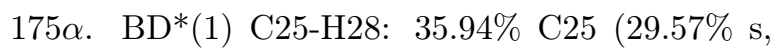

$70.34 \% \mathrm{p}) ; 64.06 \% \mathrm{H} 28(99.97 \% \mathrm{~s})$

64ß. LP(4) I50: $100 \%$ I50 (5.59\%s, $94.41 \%$ p)

176ß. BD*(1) C25-H28: $36.26 \%$ C25 (29.59\% s, $70.32 \%$ p); $63.74 \%$ H28 (99.97\% s)

\section{Dye- $\mathrm{Cl}^{+}$}

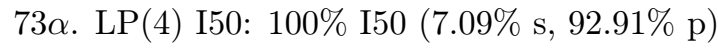

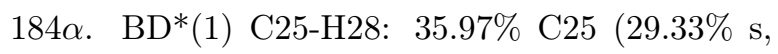
$70.58 \% \mathrm{p}) ; 64.03 \% \mathrm{H} 28(99.97 \% \mathrm{~s})$

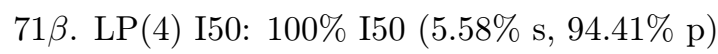
183 $\beta$. BD*(1) C25-H28: $36.27 \%$ C28 (29.36\% s, $70.55 \%$ p); $63.73 \%$ Cl45 (99.97\% s)

\section{Dye-Br ${ }^{+}$}

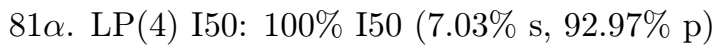
206 $\alpha$. BD*(1) C35-H38: 36.00\% C35 (29.27\% s, $70.64 \%$ p); $64.00 \%$ H38 (99.97\% s)

$79 \beta$. LP(4) I50: $100 \%$ I50 (5.70\% s, $94.30 \%$ p)

205\%. BD*(1) C35-H38: 36.29\% C35 (29.29\% s, $70.61 \%$ p); $63.71 \%$ H38 (99.97\% s)

\section{Dye-I ${ }^{+}$}

81 $\alpha$. LP(4) I50: $100 \%$ I50 (6.67\% s, $93.03 \%$ p)

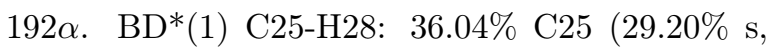
$70.71 \%$ p); $63.96 \%$ H28 (99.97\% s)

$79 \beta$. LP(4) I50: $100 \%$ I50 (5.86\% s, $94.14 \%$ p)

191ß. BD*(1) C25-H28: $36.32 \%$ C25 (29.23\% s, $70.68 \%$ p); $63.68 \%$ H28 (99.97\% s) 


\subsubsection{Amine-Iodide Interaction: Summary of Computational Analysis}

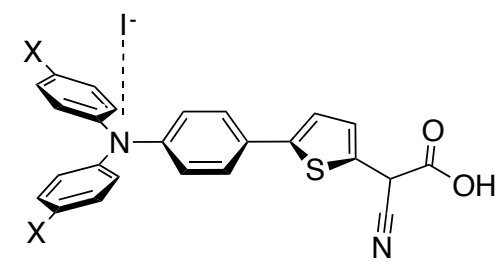

Figure S10: Schematic representation of Dye- $\mathbf{X}^{+} \ldots \mathrm{I}^{-}$iodide-amine interaction geometries (I-N).

Table S6: Calculated Halogen-Iodide Distances and Interaction Energies Describing the Interaction of Dye- $\mathbf{X}^{+}$with Iodide in the I-N interaction geometries (Figure S10). No individual second order interactions were found by NBO analysis with $E^{2}$ greater than $1.00 \mathrm{kcal} \mathrm{mol}^{-1}$.

\begin{tabular}{llll}
\hline Dye & $\begin{array}{l}\mathrm{d}_{\mathrm{N} \cdots \mathrm{I}} \\
(\AA)^{a}\end{array}$ & $\begin{array}{l}\text { van der Waals distance } \\
(\AA)^{b}\end{array}$ & $\begin{array}{l}\Delta E_{\text {int }} \\
\left.(\mathrm{kcal} \mathrm{mol})^{-1}\right)^{c}\end{array}$ \\
\hline Dye-F $^{+}$ & 3.81 & 3.61 & -5.22 \\
Dye-Cl $^{+}$ & 3.81 & 3.61 & -5.26 \\
Dye-Br $^{+}$ & 3.81 & 3.61 & -5.27 \\
Dye-I $^{+}$ & 3.81 & 3.61 & -5.24 \\
\hline
\end{tabular}

${ }^{a}$ Optimized internuclear distance between the iodide ion and the nitrogen of the triphenylamine. ${ }^{b}$ Internuclear distance between the nitrogen atom and iodide ion predicted by van der Waals radii. ${ }^{c}$ Interaction energy corrected for basis set superposition error.

A)

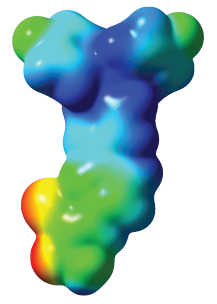

Dye- $\mathrm{F}^{+}$

B)

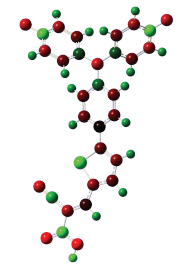

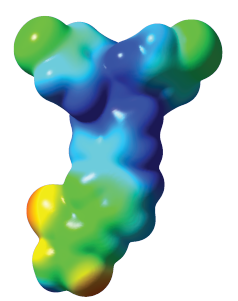
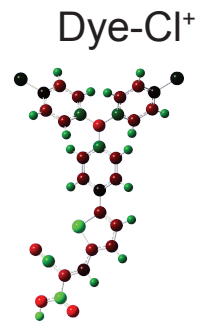
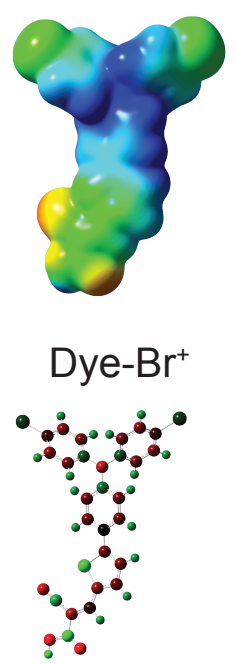
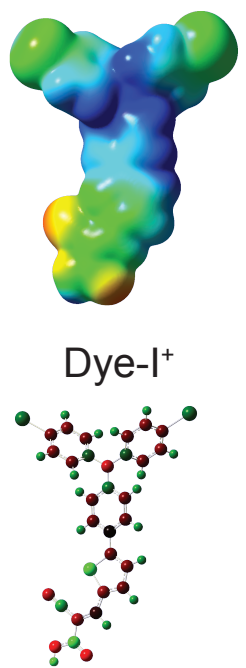

Figure S11: A) Electrostatic potential plots of Dye- $\mathbf{X}^{+}$. ESP plots are generated at iso $=0.001$ from -0.014 Hartrees (red) to +0.15 Hartrees (blue). B) Natural population analysis (NPA) plots show partial charges from -0.500 (red) to +0.500 (green). 


\subsubsection{Optimized Geometries}

Dye-F $^{+} \ldots \mathrm{I}^{-} 12 \AA$

$\mathrm{E}(\mathrm{UM} 062 \mathrm{X})=-2153.8178840600$ a.u.
Dye-Cl ${ }^{+}$. . . I- $12 \AA$

$\mathrm{E}(\mathrm{UM} 062 \mathrm{X})=-2874.5338926600 a . u$.

$\begin{array}{llll}\mathrm{C} & 8.35305700 & 0.39557100 & 0.20355300 \\ \mathrm{C} & 6.86779900 & 0.30742000 & 0.16399100 \\ \mathrm{O} & 8.94462700 & -0.75746500 & -0.11889300 \\ \mathrm{O} & 8.94826700 & 1.40395600 & 0.49600500 \\ \mathrm{C} & 6.19168600 & 1.52629900 & 0.49891900 \\ \mathrm{~N} & 5.63805100 & 2.50843800 & 0.76804200 \\ \mathrm{C} & 6.22184400 & -0.83989300 & -0.16460100 \\ \mathrm{C} & 4.81250300 & -1.10789400 & -0.25390000 \\ \mathrm{~S} & 3.53473700 & 0.03194900 & 0.04977000 \\ \mathrm{C} & 4.29721900 & -2.34576000 & -0.60917600 \\ \mathrm{C} & 2.89232900 & -2.37665300 & -0.63817500 \\ \mathrm{C} & 2.32220200 & -1.15983300 & -0.29940700 \\ \mathrm{C} & 0.90359800 & -0.83472600 & -0.22329200 \\ \mathrm{C} & 0.45238000 & 0.35076800 & 0.39551700 \\ \mathrm{C} & -0.05386100 & -1.71481600 & -0.77401100 \\ \mathrm{C} & -0.89140300 & 0.65316400 & 0.46202600 \\ \mathrm{H} & 1.16452900 & 1.03407200 & 0.85931200 \\ \mathrm{C} & -1.40116100 & -1.43213400 & -0.70563700 \\ \mathrm{H} & 0.26633800 & -2.61745700 & -1.29167800 \\ \mathrm{C} & -1.83913800 & -0.23914700 & -0.08707000 \\ \mathrm{H} & -1.22575200 & 1.55303400 & 0.97698600 \\ \mathrm{H} & -2.12529800 & -2.10183700 & -1.16819400 \\ \mathrm{~N} & -3.19682900 & 0.05559900 & -0.02004300 \\ \mathrm{C} & -4.14984900 & -0.98100000 & 0.09119400 \\ \mathrm{C} & -5.34667700 & -0.90257300 & -0.64112200 \\ \mathrm{C} & -3.89698500 & -2.07183400 & 0.94048500 \\ \mathrm{C} & -6.28257800 & -1.91825200 & -0.53276100 \\ \mathrm{H} & -5.52211900 & -0.06030900 & -1.31015400 \\ \mathrm{C} & -4.83545200 & -3.08527100 & 1.05157500 \\ \mathrm{H} & -2.98030400 & -2.10334200 & 1.52923900 \\ \mathrm{C} & -6.01077800 & -2.99197100 & 0.31133300 \\ \mathrm{H} & -7.21248600 & -1.89857600 & -1.09867300 \\ \mathrm{H} & 4.93543400 & -3.19850700 & -0.83582200 \\ \mathrm{H} & -4.67887700 & -3.93650600 & 1.71210100 \\ \mathrm{C} & -3.64253800 & 1.39492600 & -0.06242200 \\ \mathrm{C} & -4.69255800 & 1.80215900 & 0.77836400 \\ \mathrm{C} & -3.04068900 & 2.30224200 & -0.95131400 \\ \mathrm{C} & -5.13147600 & 3.11540700 & 0.73767500 \\ \mathrm{H} & -5.13762200 & 1.09353100 & 1.47664000 \\ \mathrm{C} & -3.48280500 & 3.61470900 & -0.99405400 \\ \mathrm{H} & -2.25129900 & 1.96526200 & -1.62308200 \\ \mathrm{H} & -4.51890200 & 3.99905200 & -0.14746600 \\ \mathrm{H} & -3.93057600 & 3.46929300 & 1.38700600 \\ \mathrm{H} & -6.91282100 & -3.96672400 & 0.41696600 \\ \mathrm{H} & -4.94334700 & 5.26114500 & -0.18742300 \\ \mathrm{H} & -3.26659400 & -0.87961400 \\ \mathrm{H} & -1.69649800 & -0.39915600 \\ \mathrm{H} & -0.61595700 & -0.06975400 \\ \mathrm{H} & & \\ \mathrm{H} & & \end{array}$

\begin{tabular}{|c|c|c|c|}
\hline $\mathrm{C}$ & -8.77173600 & 0.14083800 & -0.21469900 \\
\hline $\mathrm{C}$ & -7.27990600 & 0.17702400 & -0.18669200 \\
\hline 0 & -9.42394600 & -0.83235000 & 0.07266600 \\
\hline D & -9.28913900 & 1.30977100 & -0.59456000 \\
\hline 乙 & -6.62253600 & 1.39596700 & -0.55012700 \\
\hline $\mathrm{N}$ & -6.08046300 & 2.37853200 & -0.84210200 \\
\hline $\mathrm{C}$ & -6.62102500 & -0.94908200 & 0.18241200 \\
\hline $\mathrm{C}$ & -5.21139300 & -1.20038600 & 0.29542900 \\
\hline S & -3.94571100 & -0.05615600 & -0.03419800 \\
\hline $\mathrm{C}$ & -4.68616700 & -2.42174000 & 0.69492200 \\
\hline $\mathrm{C}$ & -3.28305000 & -2.43573400 & 0.73664600 \\
\hline $\mathrm{C}$ & -2.72172100 & -1.22227100 & 0.36360300 \\
\hline $\mathrm{C}$ & -1.30980400 & -0.88710400 & 0.28571900 \\
\hline $\mathrm{C}$ & -0.86875400 & 0.31500900 & -0.31345500 \\
\hline $\mathrm{C}$ & -0.33998000 & -1.77250700 & 0.81189600 \\
\hline $\mathrm{C}$ & 0.47030700 & 0.62593800 & -0.38663900 \\
\hline $\mathrm{H}$ & -1.58803700 & 1.00388800 & -0.75738800 \\
\hline $\mathrm{C}$ & 1.00286200 & -1.48223800 & 0.73590000 \\
\hline $\mathrm{H}$ & -0.64911300 & -2.68723900 & 1.31438300 \\
\hline $\mathrm{C}$ & 1.43222100 & -0.27361400 & 0.13420400 \\
\hline $\mathrm{H}$ & 0.79327400 & 1.53673500 & -0.88911200 \\
\hline $\mathrm{H}$ & 1.73363500 & -2.15698700 & 1.17987800 \\
\hline $\mathrm{N}$ & 2.78141200 & 0.02424400 & 0.05379000 \\
\hline $\mathrm{C}$ & 3.74397700 & -1.00976500 & -0.04783100 \\
\hline $\mathrm{C}$ & 4.93374200 & -0.92102400 & 0.68981800 \\
\hline $\mathrm{C}$ & 3.50647100 & -2.10036500 & -0.89765700 \\
\hline $\mathrm{C}$ & 5.87933900 & -1.93007700 & 0.58730800 \\
\hline $\mathrm{H}$ & 5.10185800 & -0.07552200 & 1.35685800 \\
\hline $\mathrm{C}$ & 4.45526000 & -3.10682900 & -1.00306400 \\
\hline $\mathrm{H}$ & 2.59410800 & -2.14156500 & -1.49280300 \\
\hline $\mathrm{C}$ & 5.63252400 & -3.01428900 & -0.25826600 \\
\hline $\mathrm{H}$ & 6.80102500 & -1.88495000 & 1.16487000 \\
\hline $\mathrm{H}$ & 4.29258700 & -3.95228800 & -1.66932200 \\
\hline $\mathrm{C}$ & 3.22849700 & 1.36769300 & 0.06457600 \\
\hline $\mathrm{C}$ & 4.25414300 & 1.76096900 & -0.80810100 \\
\hline $\mathrm{C}$ & 2.65425300 & 2.28623000 & 0.95613900 \\
\hline $\mathrm{C}$ & 4.69519500 & 3.07538600 & -0.79797600 \\
\hline $\mathrm{H}$ & 4.68344600 & 1.04255600 & -1.50643700 \\
\hline $\mathrm{C}$ & 3.09941700 & 3.59996900 & 0.96834700 \\
\hline $\mathrm{H}$ & 1.88276600 & 1.96191900 & 1.65472400 \\
\hline $\mathrm{C}$ & 4.11407200 & 3.98421900 & 0.08969700 \\
\hline $\mathrm{H}$ & 5.47882600 & 3.40090500 & -1.47996000 \\
\hline $\mathrm{H}$ & 2.67428800 & 4.32119900 & 1.66427100 \\
\hline $\mathrm{Cl}$ & 6.82052800 & -4.27162500 & -0.39143500 \\
\hline $\mathrm{Cl}$ & 4.67087200 & 5.62687900 & 0.10387700 \\
\hline $\mathrm{H}$ & -7.26389700 & -1.79791100 & 0.43230600 \\
\hline $\mathrm{H}$ & -10.25906700 & 1.22072800 & -0.59323100 \\
\hline $\mathrm{H}$ & -5.31740500 & -3.27335500 & 0.94370900 \\
\hline $\mathrm{H}$ & -2.69996100 & -3.31102800 & 1.01360200 \\
\hline & 8.50357462 & 16.99796386 & 0.19779095 \\
\hline
\end{tabular}


Dye-Br ${ }^{+} \ldots \mathrm{I}^{-} 12 \AA$

$\mathrm{E}(\mathrm{UM} 062 \mathrm{X})=-2788.1811171400$ a.u.
Dye-I ${ }^{+} \ldots \mathrm{I}^{-} 12 \AA$

$\mathrm{E}(\mathrm{UM} 062 \mathrm{X})=-2545.3616122100$ a.u.

\begin{tabular}{|c|c|c|c|}
\hline $\mathrm{C}$ & -11.32238364 & -5.71451040 & -0.22003296 \\
\hline $\mathrm{C}$ & -9.83133964 & -5.65594740 & -0.18427996 \\
\hline $\mathrm{O}$ & -11.96295664 & -6.68198740 & 0.10953104 \\
\hline $\mathrm{O}$ & -11.85304764 & -4.57253640 & -0.65886396 \\
\hline $\mathrm{C}$ & -9.18824964 & -4.44715740 & -0.60298296 \\
\hline $\mathrm{N}$ & -8.65803064 & -3.47253240 & -0.93983296 \\
\hline $\mathrm{C}$ & -9.15937164 & -6.75354540 & 0.24257604 \\
\hline $\mathrm{C}$ & -7.74699964 & -6.97823840 & 0.37585704 \\
\hline S & -6.49652264 & -5.82994640 & 0.00594104 \\
\hline $\mathrm{C}$ & -7.20646564 & -8.17347240 & 0.83076304 \\
\hline $\mathrm{C}$ & -5.80392364 & -8.16 & 6504 \\
\hline $\mathrm{C}$ & -5.25775964 & -6.9 & 2904 \\
\hline $\mathrm{C}$ & -3.85101364 & -6.60 & 19104 \\
\hline $\mathrm{C}$ & -2.86908564 & -7.46 & 34804 \\
\hline $\mathrm{C}$ & -3.42546464 & -5.4 & -0.24093296 \\
\hline $\mathrm{C}$ & -1.53048264 & -7.1 & 0.83801304 \\
\hline $\mathrm{H}$ & -3.16532064 & -8.3 & 604 \\
\hline $\mathrm{C}$ & -2.09096964 & -5.0 & 6696 \\
\hline $\mathrm{H}$ & -4.15349364 & -4.7 & 4096 \\
\hline $\mathrm{C}$ & -1.11680164 & -5.9 & 4904 \\
\hline $\mathrm{H}$ & -0.79084 & -7.8 & 7804 \\
\hline $\mathrm{H}$ & -1.78 & -4.1 & 9696 \\
\hline $\mathrm{N}$ & 0.22 & -5.6 & 0.12 \\
\hline $\mathrm{C}$ & 0.65 & & 504 \\
\hline $\mathrm{C}$ & $1.67990^{\prime}$ & & 9396 \\
\hline $\mathrm{C}$ & 0.07361 & -3. & \\
\hline C & 2.10 & -2. & 896 \\
\hline $\mathrm{H}$ & 2.118370 & -4.6 & 8396 \\
\hline $\mathrm{C}$ & 0.50259 & -2. & 704 \\
\hline $\mathrm{H}$ & -0.69407 & & \\
\hline $\mathrm{C}$ & 1.51311636 & -1.6 & 2104 \\
\hline $\mathrm{H}$ & 2.88 & -2 & 196 \\
\hline $\mathrm{H}$ & 0.06418236 & -1.3 & 1804 \\
\hline $\mathrm{C}$ & 1.20224536 & -6.6 & 3104 \\
\hline $\mathrm{C}$ & 2.38996636 & -6.5 & 0604 \\
\hline $\mathrm{C}$ & & -7.7 & 2696 \\
\hline $\mathrm{C}$ & 3.34 & -7.5 & 7904 \\
\hline $\mathrm{H}$ & 2.55016636 & -5.7 & 3604 \\
\hline $\mathrm{C}$ & 1.93304636 & -8.7 & -0.87997396 \\
\hline $\mathrm{H}$ & 0.06453136 & -7.8 & -1.38748296 \\
\hline $\mathrm{C}$ & 3.10784536 & -8.6 & -0.13403496 \\
\hline $\mathrm{H}$ & 4.26318136 & -7.49599840 & 1.27497504 \\
\hline $\mathrm{H}$ & 1.77268136 & -9.6 & -1.53442896 \\
\hline $\mathrm{Br}$ & 2.09903636 & 0.13044060 & 0.08685704 \\
\hline $\mathrm{Br}$ & 4.41287836 & -10.02966140 & -0.25089696 \\
\hline $\mathrm{H}$ & -9.79184864 & -7.59867140 & 0.52879604 \\
\hline $\mathrm{H}$ & -12.82164864 & -4.67512240 & -0.65998196 \\
\hline $\mathrm{H}$ & -7.82677864 & -9.02298940 & 1.11193204 \\
\hline $\mathrm{H}$ & -5.21036064 & -9.01803140 & 1.20055104 \\
\hline I & 5.79294901 & 11.54746007 & 0.00515209 \\
\hline
\end{tabular}

$\begin{array}{llll}\mathrm{C} & -10.12222800 & -0.34646800 & -0.51171600 \\ \mathrm{C} & -8.63824600 & -0.27555400 & -0.46563400 \\ \mathrm{O} & -10.78979800 & -1.05032800 & 0.20849800 \\ \mathrm{O} & -10.63404600 & 0.46178600 & -1.44506000 \\ \mathrm{C} & -7.97067200 & 0.60214000 & -1.37730500 \\ \mathrm{~N} & -7.42233800 & 1.31130400 & -2.11371700 \\ \mathrm{C} & -7.98665300 & -1.04937000 & 0.44411600 \\ \mathrm{C} & -6.59060600 & -1.18715900 & 0.71549800 \\ \mathrm{~S} & -5.29551200 & -0.35618900 & -0.10979200 \\ \mathrm{C} & -6.08053300 & -2.02193700 & 1.69790100 \\ \mathrm{C} & -4.67663900 & -1.99034400 & 1.78992600 \\ \mathrm{C} & -4.09497900 & -1.12850300 & 0.87631200 \\ \mathrm{C} & -2.66889800 & -0.84622800 & 0.68644500 \\ \mathrm{C} & -2.22692200 & 0.27278300 & -0.03743900 \\ \mathrm{C} & -1.69725800 & -1.69953100 & 1.23591700 \\ \mathrm{C} & -0.87488100 & 0.52905500 & -0.21314500 \\ \mathrm{H} & -2.94954200 & 0.96272600 & -0.47730900 \\ \mathrm{C} & -0.34355000 & -1.44118900 & 1.07985800 \\ \mathrm{H} & -1.99990600 & -2.58419200 & 1.79639800 \\ \mathrm{C} & 0.08916800 & -0.32270800 & 0.34886900 \\ \mathrm{H} & -0.55919100 & 1.39954400 & -0.78811200 \\ \mathrm{H} & 0.38995700 & -2.11329900 & 1.52535700 \\ \mathrm{~N} & 1.46243100 & -0.05917500 & 0.18298000 \\ \mathrm{C} & 2.39047400 & -1.12300700 & 0.06157300 \\ \mathrm{C} & 3.61969000 & -1.06343600 & 0.72961800 \\ \mathrm{C} & 2.09555800 & -2.23735200 & -0.73393900 \\ \mathrm{C} & 4.54489500 & -2.09564300 & 0.60040200 \\ \mathrm{H} & 3.85480500 & -0.20036900 & 1.35361200 \\ \mathrm{C} & 3.00813500 & -3.28199700 & -0.85202100 \\ \mathrm{H} & -8.58847100 & 5.88432000 & -0.00705900 \\ \mathrm{H} & -1.14381900 & -2.28745100 & -1.26428600 \\ \mathrm{I} & -6.72459300 & -2.62959600 & 2.33267600 \\ \mathrm{H} & 4.23152500 & -3.20307500 & -0.18692800 \\ \mathrm{H} & 5.49791700 & -2.03603900 & 1.12494300 \\ \mathrm{H} & 2.76613400 & -4.14282100 & -1.47440300 \\ \mathrm{C} & 1.93455900 & 1.27684300 & 0.13879700 \\ \mathrm{C} & 2.89586900 & 1.65282800 & -0.80719400 \\ \mathrm{C} & 1.45293300 & 2.23023400 & 1.04422100 \\ \mathrm{C} & 3.37566500 & 2.95932500 & -0.84586000 \\ \mathrm{H} & 3.27270500 & 0.91581700 & -1.51744500 \\ \mathrm{C} & 1.91415400 & 3.54286700 & 0.99887800 \\ \mathrm{H} & 0.71008400 & 1.94250100 & 1.78923400 \\ \mathrm{H} & 2.87748800 & 3.89950300 & 0.05535000 \\ \mathrm{H} & 1.53134900 & 4.27504600 & 1.70893200 \\ \mathrm{H} & -2.56576100 & 2.51620400 \\ \mathrm{H} & 17.18313345 & -0.35369508\end{array}$


Dye- $\mathbf{F}^{+} \ldots \mathrm{I}^{-}$Interaction at halogen

$\mathrm{E}(\mathrm{UM} 062 \mathrm{X})=-2153.8188376900$ a.u.

BSSE Energy $=0.0000301573$ a.u.

BSSE Corrected E(UM062X) $=-2153.8188075327$ a.u.
Dye- $\mathbf{C l}^{+} \ldots \mathrm{I}^{-}$Interaction at halogen

$\mathrm{E}(\mathrm{UM} 062 \mathrm{X})=-2874.5358721500$ a.u.

BSSE Energy $=0.0000681130$ a.u.

BSSE Corrected E(UM062X) $=-2874.5358040370$ a.u.

\begin{tabular}{|c|c|c|c|}
\hline $\mathrm{C}$ & -8.93110700 & -3.70756900 & 0.22763900 \\
\hline $\mathrm{H}$ & 1.08838500 & 0.27043800 & -1.59043600 \\
\hline $\mathrm{C}$ & -7.69253200 & -2.88293500 & 0.18262700 \\
\hline $\mathrm{C}$ & 4.02511500 & -0.30126300 & -0.01433000 \\
\hline $\mathrm{O}$ & -10.01604000 & -3.02552100 & -0.14745400 \\
\hline $\mathrm{H}$ & 4.91909300 & 0.88388500 & 1.54431600 \\
\hline $\mathrm{O}$ & -8.94210300 & -4.86562300 & 0.56715200 \\
\hline $\mathrm{H}$ & 2.99272900 & -1.35947400 & -1.57832500 \\
\hline $\mathrm{C}$ & -6.50258300 & -3.57831400 & 0.57674500 \\
\hline $\mathrm{F}$ & 2.03165800 & 7.76542700 & 0.40427700 \\
\hline $\mathrm{N}$ & -5.53686000 & -4.13534100 & 0.89388700 \\
\hline $\mathrm{F}$ & 5.04541500 & -1.17196300 & -0.01791200 \\
\hline $\mathrm{C}$ & -7.70556300 & -1.58237800 & -0.20441200 \\
\hline $\mathrm{H}$ & -7.76516500 & 1.07175200 & -0.99685500 \\
\hline $\mathrm{C}$ & -6.62081900 & -0.64508200 & -0.31068100 \\
\hline $\mathrm{H}$ & -5.53741500 & 2.44699400 & -1.05009100 \\
\hline S & -4.95109600 & -0.97159100 & 0.04673700 \\
\hline $\mathrm{H}$ & -8.68074900 & -1.17499400 & -0.47998900 \\
\hline $\mathrm{C}$ & -6.79052000 & 0.66710900 & -0.72847000 \\
\hline $\mathrm{H}$ & -10.77558600 & -3.63165200 & -0.09166800 \\
\hline $\mathrm{C}$ & -5.59240500 & 1.39993200 & -0.76133600 \\
\hline I & 8.51724000 & -3.75562600 & -0.05768300 \\
\hline $\mathrm{C}$ & -4.49435200 & 0.65224300 & -0.36392100 \\
\hline $\mathrm{C}$ & -3.10897600 & 1.09010400 & -0.27208200 \\
\hline $\mathrm{C}$ & -2.12527600 & 0.29469800 & 0.35590600 \\
\hline $\mathrm{C}$ & -2.72200200 & 2.33653300 & -0.81448500 \\
\hline $\mathrm{C}$ & -0.81560900 & 0.71468000 & 0.44104400 \\
\hline $\mathrm{H}$ & -2.39717200 & -0.65785100 & 0.81163300 \\
\hline $\mathrm{C}$ & -1.41961700 & 2.77592200 & -0.72711300 \\
\hline $\mathrm{H}$ & -3.44808800 & 2.95563700 & -1.33838800 \\
\hline $\mathrm{C}$ & -0.44350700 & 1.96846600 & -0.09712800 \\
\hline $\mathrm{H}$ & -0.07805500 & 0.10674600 & 0.96346200 \\
\hline $\mathrm{H}$ & -1.12961000 & 3.72215700 & -1.18221400 \\
\hline $\mathrm{N}$ & 0.87025000 & 2.40537200 & -0.00591600 \\
\hline C & 1.16328500 & 3.78565100 & 0.09593300 \\
\hline $\mathrm{C}$ & 2.24187300 & 4.31786300 & -0.62918100 \\
\hline $\mathrm{C}$ & 0.38441100 & 4.60241900 & 0.93196400 \\
\hline $\mathrm{C}$ & 2.53474100 & 5.66898900 & -0.52791500 \\
\hline $\mathrm{H}$ & 2.82760900 & 3.67483300 & -1.28586000 \\
\hline $\mathrm{C}$ & 0.67835300 & 5.95339100 & 1.03724200 \\
\hline $\mathrm{H}$ & -0.42770700 & 4.16906600 & 1.51570800 \\
\hline $\mathrm{C}$ & 1.74615300 & 6.45760700 & 0.30320300 \\
\hline $\mathrm{H}$ & 3.35336600 & 6.11803300 & -1.08766700 \\
\hline $\mathrm{H}$ & 0.10522100 & 6.61206200 & 1.68745800 \\
\hline $\mathrm{C}$ & 1.93967100 & 1.48050600 & -0.00727000 \\
\hline $\mathrm{C}$ & 3.02091400 & 1.67507300 & 0.86832400 \\
\hline $\mathrm{C}$ & 1.91655500 & 0.38929800 & -0.89181000 \\
\hline C & 4.07315500 & 0.77324800 & 0.86807900 \\
\hline $\mathrm{H}$ & 3.01684200 & 2.51663500 & 1.56085700 \\
\hline $\mathrm{C}$ & 2.97028600 & -0.51166600 & -0.89576800 \\
\hline
\end{tabular}

\begin{tabular}{|c|c|c|c|}
\hline $\mathrm{C}$ & -9.20395100 & -3.63277300 & 0.20122300 \\
\hline & -7.89542300 & -2.91518200 & 0.18276500 \\
\hline & -10.25389400 & -3.12033800 & -0.09911000 \\
\hline J & -9.06785700 & -4.90185400 & 0.58781300 \\
\hline & -6.71910500 & -3.63722600 & 0.56350000 \\
\hline & -5.76096800 & -4.21399000 & 0.86981800 \\
\hline C & -7.88636800 & -1.61338000 & -0.19665900 \\
\hline $\mathrm{C}$ & -6.79128900 & -0.69073400 & -0.30802200 \\
\hline & -5.12556900 & -1.04469200 & 0.03805200 \\
\hline $\mathrm{C}$ & -6.94457400 & 0.62590200 & -0.72083100 \\
\hline $\mathrm{C}$ & -5.73652100 & 1.33977400 & -0.75934700 \\
\hline $\mathrm{C}$ & -4.64630200 & 0.57368800 & -0.37053000 \\
\hline $\mathrm{C}$ & -3.25686300 & 0.99058000 & -0.28531500 \\
\hline $\mathrm{C}$ & -2.27759400 & 0.17253900 & 0.32340000 \\
\hline $\mathrm{C}$ & -2.85653000 & 2.24027700 & -0.81410200 \\
\hline $\mathrm{C}$ & -0.96298200 & 0.57295200 & 0.40324600 \\
\hline $\mathrm{H}$ & -2.55899300 & -0.78204600 & 0.76894500 \\
\hline $\mathrm{C}$ & -1.54886000 & 2.66009600 & -0.73236600 \\
\hline $\mathrm{H}$ & -3.57824200 & 2.87639700 & -1.32328800 \\
\hline $\mathrm{C}$ & -0.57638500 & 1.83054000 & -0.12123300 \\
\hline $\mathrm{H}$ & -0.23048200 & -0.05172800 & 0.91265300 \\
\hline $\mathrm{H}$ & -1.25024400 & 3.60782600 & -1.17844200 \\
\hline $\mathrm{N}$ & 0.74004800 & 2.24730700 & -0.03525500 \\
\hline $\mathrm{C}$ & 1.05675100 & 3.62581200 & 0.05364200 \\
\hline $\mathrm{C}$ & 2.13026500 & 4.13597500 & -0.69082400 \\
\hline $\mathrm{C}$ & 0.30715300 & 4.45920700 & 0.89664900 \\
\hline $\mathrm{C}$ & 2.44552900 & 5.48373200 & -0.60261700 \\
\hline $\mathrm{H}$ & 2.69743600 & 3.48102600 & -1.35218200 \\
\hline $\mathrm{C}$ & 0.62547500 & 5.80658700 & 0.98816100 \\
\hline $\mathrm{H}$ & -0.50278400 & 4.04465800 & 1.49708500 \\
\hline $\mathrm{C}$ & 1.68972500 & 6.30548200 & 0.23575500 \\
\hline $\mathrm{H}$ & 3.26553100 & 5.89978000 & -1.18559700 \\
\hline $\mathrm{H}$ & 0.06305900 & 6.46467600 & 1.64841500 \\
\hline $\mathrm{C}$ & 1.79970500 & 1.30860900 & -0.03037200 \\
\hline $\mathrm{C}$ & 2.87919200 & 1.49217300 & 0.84710400 \\
\hline $\mathrm{C}$ & 1.76999100 & 0.21792200 & -0.91275500 \\
\hline $\mathrm{C}$ & 3.92131400 & 0.57789100 & 0.85123100 \\
\hline $\mathrm{H}$ & 2.88472400 & 2.33527400 & 1.53793700 \\
\hline $\mathrm{C}$ & 2.81578000 & -0.69328400 & -0.91084600 \\
\hline $\mathrm{H}$ & 0.94436500 & 0.10503300 & -1.61558200 \\
\hline $\mathrm{C}$ & 3.88150500 & -0.50746900 & -0.02784900 \\
\hline $\mathrm{H}$ & 4.75843400 & 0.69661600 & 1.53736300 \\
\hline $\mathrm{H}$ & 2.81545700 & -1.53654600 & -1.59972600 \\
\hline $\mathrm{Cl}$ & 2.08855800 & 7.99299100 & 0.34941200 \\
\hline $\mathrm{Cl}$ & 5.19094800 & -1.64873400 & -0.02700400 \\
\hline $\mathrm{H}$ & -8.86584000 & -1.20360300 & -0.45918000 \\
\hline $\mathrm{H}$ & -9.95080000 & -5.31285300 & 0.57776100 \\
\hline $\mathrm{H}$ & -7.91498300 & 1.04540400 & -0.98131900 \\
\hline $\mathrm{H}$ & -5.66726300 & 2.38658400 & -1.04583000 \\
\hline & 7.98020300 & -4.18165300 & -0.03695100 \\
\hline
\end{tabular}


Dye-Br ${ }^{+} \ldots \mathrm{I}^{-}$Interaction at halogen $\mathrm{E}(\mathrm{UM} 062 \mathrm{X})=-2788.1848546700$ a.u. BSSE Energy $=0.0000967712$ a.u.

BSSE Corrected E(UM062X) $=-2788.1847578988$ a.u.
Dye- $\mathbf{I}^{+} \ldots \mathrm{I}^{-}$Interaction at halogen

$\mathrm{E}(\mathrm{UM} 062 \mathrm{X})=-2545.3674654300$ a.u. BSSE Energy $=0.0000680305$ a.u.

BSSE Corrected E(UM062X) $=-2545.3673973995$ a.u.

\begin{tabular}{|c|c|c|c|}
\hline $\mathrm{C}$ & 9.64921400 & -3.83439700 & -0.23777200 \\
\hline $\mathrm{C}$ & 8.33268000 & -3.13210700 & -0.21020600 \\
\hline $\mathrm{O}$ & 10.69255700 & -3.31576900 & 0.07476400 \\
\hline $\mathrm{O}$ & 9.52868000 & -5.09773600 & -0.64780300 \\
\hline $\mathrm{C}$ & 7.16549400 & -3.86090700 & -0.60590300 \\
\hline $\mathrm{N}$ & 6.21479900 & -4.44349000 & -0.92418900 \\
\hline $\mathrm{C}$ & 8.30809200 & -1.83688700 & 0.19061600 \\
\hline $\mathrm{C}$ & 7.20254000 & -0.92843400 & 0.31421100 \\
\hline S & 5.54142300 & -1.29677200 & -0.03923200 \\
\hline $\mathrm{C}$ & 7.33973200 & 0.38369500 & 0.74632100 \\
\hline $\mathrm{C}$ & 6.12310800 & 1.08273800 & 0.79338800 \\
\hline $\mathrm{C}$ & 5.04264800 & 0.30961400 & 0.39217600 \\
\hline $\mathrm{C}$ & 3.64774300 & 0.71054100 & 0.31185100 \\
\hline $\mathrm{C}$ & 3.23154800 & 1.94753400 & 0.85729500 \\
\hline $\mathrm{C}$ & 2.67945900 & -0.11138900 & -0.30837300 \\
\hline $\mathrm{C}$ & 1.91818200 & 2.35146000 & 0.78138400 \\
\hline $\mathrm{H}$ & 3.94529000 & 2.58619800 & 1.37451700 \\
\hline $\mathrm{C}$ & 1.35949100 & 0.27302400 & -0.38246400 \\
\hline $\mathrm{H}$ & 2.97307500 & -1.05596400 & -0.76720200 \\
\hline $\mathrm{C}$ & 0.95687000 & 1.51747500 & 0.15976600 \\
\hline $\mathrm{H}$ & 1.60742200 & 3.28949500 & 1.23959900 \\
\hline $\mathrm{H}$ & 0.63486100 & -0.35396500 & -0.90025500 \\
\hline $\mathrm{N}$ & -0.36617700 & 1.91771300 & 0.08032600 \\
\hline $\mathrm{C}$ & -1.41194900 & 0.96496600 & 0.06947000 \\
\hline $\mathrm{C}$ & -2.49918200 & 1.14314700 & -0.79983200 \\
\hline $\mathrm{C}$ & -1.36068400 & -0.13766900 & 0.93625600 \\
\hline $\mathrm{C}$ & -3.52527200 & 0.21039000 & -0.81304300 \\
\hline $\mathrm{H}$ & -2.52197500 & 1.99562500 & -1.47887600 \\
\hline $\mathrm{C}$ & -2.39106300 & -1.06651800 & 0.92455500 \\
\hline $\mathrm{H}$ & -0.53043800 & -0.24630200 & 1.63445800 \\
\hline $\mathrm{C}$ & -3.46514200 & -0.88966400 & 0.04804200 \\
\hline $\mathrm{H}$ & -4.36424100 & 0.33040400 & -1.49690000 \\
\hline $\mathrm{H}$ & -2.36694600 & -1.91772600 & 1.60353900 \\
\hline $\mathrm{C}$ & -0.70005400 & 3.29265400 & 0.00257000 \\
\hline $\mathrm{C}$ & -1.77826900 & 3.78372700 & 0.75275700 \\
\hline $\mathrm{C}$ & 0.03786700 & 4.14170000 & -0.83484700 \\
\hline $\mathrm{C}$ & -2.11062300 & 5.12859200 & 0.67536600 \\
\hline $\mathrm{H}$ & -2.33600900 & 3.11683200 & 1.41036900 \\
\hline $\mathrm{C}$ & -0.29767900 & 5.48608500 & -0.91595600 \\
\hline $\mathrm{H}$ & 0.85191100 & 3.74191900 & -1.43992500 \\
\hline $\mathrm{C}$ & -1.36712100 & 5.96721500 & -0.15825700 \\
\hline $\mathrm{H}$ & -2.93590100 & 5.52419100 & 1.26533800 \\
\hline $\mathrm{H}$ & 0.25892500 & 6.15118400 & -1.57428500 \\
\hline $\mathrm{Br}$ & -4.86734800 & -2.16679900 & 0.02913700 \\
\hline $\mathrm{Br}$ & -1.82605100 & 7.80115200 & -0.26854500 \\
\hline $\mathrm{H}$ & 9.28236700 & -1.42054500 & 0.46204100 \\
\hline $\mathrm{H}$ & 10.41629900 & -5.49861000 & -0.64272000 \\
\hline $\mathrm{H}$ & 8.30486100 & 0.81064500 & 1.01428700 \\
\hline $\mathrm{H}$ & 6.04083300 & 2.12432200 & 1.09513300 \\
\hline I & -7.52622900 & -4.67967400 & 0.01062300 \\
\hline
\end{tabular}

\begin{tabular}{|c|c|c|c|}
\hline & 9.95237300 & -4.31505100 & \\
\hline & 8.65059200 & -3.58616800 & \\
\hline & 10.99620300 & -3.83927900 & \\
\hline & 9.81863800 & -5.54916300 & -0.76707900 \\
\hline & & & \\
\hline & .52891400 & & \\
\hline & 8.64103600 & -2.31567400 & 179800 \\
\hline & 7.55257700 & -1.38993800 & \\
\hline & 5.89279600 & -1.70019200 & -0.04376200 \\
\hline & & & \\
\hline & 6.50713300 & 0 & \\
\hline & & & \\
\hline & & 0 & \\
\hline & & & 28200 \\
\hline & & & \\
\hline & & 00 & \\
\hline & & & \\
\hline & & 2.00 & \\
\hline & & & \\
\hline & & & \\
\hline & & 00 & -0 . \\
\hline & & & \\
\hline & & & \\
\hline & & & \\
\hline & & & \\
\hline & 0.53 & 0 & \\
\hline & & & \\
\hline & & & \\
\hline & & & \\
\hline & & & \\
\hline & -0.83456 & 0 & \\
\hline & -2.43 & & \\
\hline & & & \\
\hline & & & \\
\hline & & & \\
\hline & & & \\
\hline & -3.14937900 & 0 & 400 \\
\hline & -2.10242700 & 0 & 07700 \\
\hline & -2.0 & -1.3 & \\
\hline & -0.15738900 & -0.5 & \\
\hline & & -1.0 & \\
\hline & -3.98444900 & 0.18 & -1.48277900 \\
\hline & -2.03100800 & -2.1 & 99100 \\
\hline & & & -0.23006700 \\
\hline & -4.72626000 & -2.45601600 & 0.02738000 \\
\hline & & & \\
\hline & 10.69751900 & -5.96879100 & -0.76057800 \\
\hline & 8.67567000 & 0.28474000 & 1.18131700 \\
\hline & & & \\
\hline & -7.52341300 & -4.84436800 & 0.0034240 \\
\hline
\end{tabular}


Dye- $\mathbf{F}^{+} \ldots \mathrm{I}^{-}$Interaction at phenyl

$\mathrm{E}(\mathrm{UM} 062 \mathrm{X})=-2153.8241349000$ a.u.

BSSE Energy $=0.0001914976$ a.u.

BSSE Corrected $\mathrm{E}(\mathrm{UM} 062 \mathrm{X})=-2153.8239434024$ a.u.
Dye- $\mathrm{Cl}^{+} \ldots \mathrm{I}^{-}$Interaction at phenyl

$\mathrm{E}(\mathrm{UM} 062 \mathrm{X})=-2874.5403221800 a . u$.

BSSE Energy $=0.0002015505$ a.u.

BSSE Corrected E(UM062X) $=-2874.5401206295$ a.u.

\begin{tabular}{|c|c|c|c|}
\hline $\mathrm{C}$ & -9.56543000 & -0.95515100 & -0.19709900 \\
\hline $\mathrm{C}$ & -8.10124700 & -0.70854300 & -0.10137100 \\
\hline $\mathrm{O}$ & -10.21361700 & 0.03304800 & -0.81956900 \\
\hline $\mathrm{O}$ & -10.10086500 & -1.94368700 & 0.24245300 \\
\hline $\mathrm{C}$ & -7.36614600 & -1.74648900 & 0.55981800 \\
\hline $\mathrm{N}$ & -6.76627500 & -2.58245100 & 1.09361500 \\
\hline $\mathrm{C}$ & -7.52430800 & 0.41267500 & -0.60386700 \\
\hline $\mathrm{C}$ & -6.14482700 & 0.81494200 & -0.60574700 \\
\hline S & -4.81527900 & -0.08993100 & 0.05793900 \\
\hline $\mathrm{C}$ & -5.70261100 & 2.00634500 & -1.16042800 \\
\hline $\mathrm{C}$ & -4.31219400 & 2.18853200 & -1.05431800 \\
\hline $\mathrm{C}$ & -3.68133100 & 1.13607900 & -0.41253900 \\
\hline $\mathrm{C}$ & -2.25625100 & 0.99195100 & -0.13069900 \\
\hline $\mathrm{C}$ & -1.78161400 & 0.02939100 & 0.78375100 \\
\hline $\mathrm{C}$ & -1.32115700 & 1.82698600 & -0.77814800 \\
\hline $\mathrm{C}$ & -0.43210100 & -0.10308700 & 1.04281700 \\
\hline $\mathrm{H}$ & -2.48211800 & -0.60815900 & 1.32412800 \\
\hline $\mathrm{C}$ & 0.02967600 & 1.71464000 & -0.52013600 \\
\hline $\mathrm{H}$ & -1.65470600 & 2.55221500 & -1.51861300 \\
\hline $\mathrm{C}$ & 0.49047900 & 0.74347700 & 0.39378700 \\
\hline $\mathrm{H}$ & -0.08233600 & -0.82692400 & 1.77810100 \\
\hline $\mathrm{H}$ & 0.74393900 & 2.34135500 & -1.05306900 \\
\hline $\mathrm{N}$ & 1.85663000 & 0.62038200 & 0.65445500 \\
\hline $\mathrm{C}$ & 2.68636300 & 1.76170200 & 0.65501300 \\
\hline $\mathrm{C}$ & 3.97663700 & 1.67371000 & 0.10412500 \\
\hline $\mathrm{C}$ & 2.21915500 & 2.96522000 & 1.21253100 \\
\hline $\mathrm{C}$ & 4.79594900 & 2.79166000 & 0.10295300 \\
\hline $\mathrm{H}$ & 4.32394800 & 0.73820000 & -0.34199300 \\
\hline $\mathrm{C}$ & 3.04034800 & 4.08145900 & 1.21441900 \\
\hline $\mathrm{H}$ & 1.22949300 & 3.01007900 & 1.66681300 \\
\hline $\mathrm{C}$ & 4.31077400 & 3.97116100 & 0.65755700 \\
\hline $\mathrm{H}$ & 5.79483600 & 2.76219200 & -0.33024900 \\
\hline $\mathrm{H}$ & 2.71854800 & 5.02479800 & 1.65219300 \\
\hline $\mathrm{C}$ & 2.42065000 & -0.64436900 & 0.91485300 \\
\hline C & 3.42234200 & -0.76966500 & 1.89445300 \\
\hline $\mathrm{C}$ & 1.98106600 & -1.76658900 & 0.18934700 \\
\hline $\mathrm{C}$ & 3.97789000 & -2.01156100 & 2.15090800 \\
\hline $\mathrm{H}$ & 3.73608100 & 0.10276400 & 2.46715900 \\
\hline $\mathrm{C}$ & 2.53832600 & -3.00873600 & 0.44336100 \\
\hline $\mathrm{H}$ & 1.22937400 & -1.65081400 & -0.59127700 \\
\hline $\mathrm{C}$ & 3.52677600 & -3.10379500 & 1.41684200 \\
\hline $\mathrm{H}$ & 4.74488900 & -2.14703900 & 2.91156100 \\
\hline $\mathrm{H}$ & 2.23637000 & -3.89389900 & -0.11385500 \\
\hline $\mathrm{F}$ & 5.10473300 & 5.05365600 & 0.65964300 \\
\hline $\mathrm{F}$ & 4.07400800 & -4.30489200 & 1.65750500 \\
\hline $\mathrm{H}$ & -6.38204800 & 2.71987800 & -1.62425500 \\
\hline $\mathrm{H}$ & -3.79181000 & 3.07185700 & -1.41767400 \\
\hline $\mathrm{H}$ & -8.20059300 & 1.12444900 & -1.08253400 \\
\hline $\mathrm{H}$ & -11.15708100 & -0.20507900 & -0.83940900 \\
\hline & 5.86696400 & -1.43675300 & -1.53435900 \\
\hline
\end{tabular}

\begin{tabular}{|c|c|c|c|}
\hline $\mathrm{C}$ & -9.86719600 & -0.72718700 & -0.24994800 \\
\hline & -8.38743700 & -0.59957600 & -0.11156700 \\
\hline & -10.56910600 & 0.11417900 & -0.75509200 \\
\hline J & -10.31537600 & -1.87950100 & 0.25093900 \\
\hline & -7.66725500 & -1.67224900 & 0.50541900 \\
\hline & -7.07510600 & -2.53570200 & 1.00386800 \\
\hline $\mathrm{C}$ & -7.79742000 & 0.52908000 & -0.57804300 \\
\hline $\mathrm{C}$ & -6.41529000 & 0.91769600 & -0.58072400 \\
\hline & -5.09212500 & -0.02414300 & 0.04032200 \\
\hline $\mathrm{C}$ & -5.96472600 & 2.12239400 & -1.10056200 \\
\hline $\mathrm{C}$ & -4.57234000 & 2.28466100 & -1.00190900 \\
\hline $\mathrm{C}$ & -3.94715900 & 1.20350500 & -0.40048600 \\
\hline $\mathrm{C}$ & -2.52366100 & 1.03421200 & -0.13834400 \\
\hline $\mathrm{C}$ & -2.04859700 & 0.01417500 & 0.71379200 \\
\hline $\mathrm{C}$ & -1.58538000 & 1.90019600 & -0.74226600 \\
\hline $\mathrm{C}$ & -0.69982500 & -0.14451200 & 0.95270200 \\
\hline $\mathrm{H}$ & -2.74977200 & -0.64926300 & 1.22106300 \\
\hline $\mathrm{C}$ & -0.23498600 & 1.76182700 & -0.50373800 \\
\hline $\mathrm{H}$ & -1.91778800 & 2.67130600 & -1.43512700 \\
\hline $\mathrm{C}$ & 0.22747200 & 0.73215800 & 0.34665800 \\
\hline $\mathrm{H}$ & -0.35130400 & -0.91314200 & 1.64146700 \\
\hline $\mathrm{H}$ & 0.47931300 & 2.41237800 & -1.00703300 \\
\hline $\mathrm{N}$ & 1.59009500 & 0.58034000 & 0.58344300 \\
\hline $\mathrm{C}$ & 2.44359100 & 1.70644400 & 0.60533500 \\
\hline $\mathrm{C}$ & 3.71673500 & 1.61407600 & 0.02105200 \\
\hline $\mathrm{C}$ & 2.01635300 & 2.89596600 & 1.21765700 \\
\hline $\mathrm{C}$ & 4.55701000 & 2.71700900 & 0.03853100 \\
\hline $\mathrm{H}$ & 4.03865600 & 0.68863300 & -0.46376500 \\
\hline $\mathrm{C}$ & 2.86031900 & 3.99595400 & 1.23774000 \\
\hline $\mathrm{H}$ & 1.03977500 & 2.94515600 & 1.69928900 \\
\hline $\mathrm{C}$ & 4.12103900 & 3.89665500 & 0.64499300 \\
\hline $\mathrm{H}$ & 5.54160100 & 2.66700900 & -0.42473800 \\
\hline $\mathrm{H}$ & 2.55234700 & 4.92159000 & 1.72113500 \\
\hline $\mathrm{C}$ & 2.14185600 & -0.70205100 & 0.79278000 \\
\hline $\mathrm{C}$ & 3.14399500 & -0.87575200 & 1.76141500 \\
\hline $\mathrm{C}$ & 1.69301600 & -1.78796200 & 0.02312300 \\
\hline $\mathrm{C}$ & 3.69102400 & -2.13228900 & 1.96272900 \\
\hline $\mathrm{H}$ & 3.47011300 & -0.03104400 & 2.36795300 \\
\hline $\mathrm{C}$ & 2.24397200 & -3.04383000 & 0.22271600 \\
\hline $\mathrm{H}$ & 0.94061000 & -1.63607800 & -0.75078100 \\
\hline $\mathrm{C}$ & 3.23848900 & -3.20259800 & 1.18861500 \\
\hline $\mathrm{H}$ & 4.46074300 & -2.28733200 & 2.71697300 \\
\hline $\mathrm{H}$ & 1.92328200 & -3.89158800 & -0.38044200 \\
\hline $\mathrm{Cl}$ & 5.17558100 & 5.27751700 & 0.66701000 \\
\hline $\mathrm{Cl}$ & 3.94689200 & -4.77043200 & 1.42177200 \\
\hline $\mathrm{H}$ & -8.48308700 & 1.25617300 & -1.02254500 \\
\hline $\mathrm{H}$ & -11.28156200 & -1.90062000 & 0.12997500 \\
\hline $\mathrm{H}$ & -6.64048200 & 2.85777100 & -1.53447800 \\
\hline $\mathrm{H}$ & -4.04550100 & 3.17313400 & -1.34242900 \\
\hline & 5.59593100 & -1.49690800 & -1.64782900 \\
\hline
\end{tabular}


Dye-Br ${ }^{+} \ldots \mathrm{I}^{-}$Interaction at phenyl

$\mathrm{E}(\mathrm{UM} 062 \mathrm{X})=-2788.1875967200$ a.u.

BSSE Energy $=0.0002020101$ a.u.

BSSE Corrected E(UM062X) $=-2788.1873947099$ a.u.
Dye-I ${ }^{+} \ldots \mathrm{I}^{-}$Interaction at phenyl

$\mathrm{E}(\mathrm{UM} 062 \mathrm{X})=-2545.3681357400$ a.u.

BSSE Energy $=0.0001919397$ a.u.

BSSE Corrected E(UM062X) $=-2545.3679438003$ a.u.

\begin{tabular}{|c|c|c|c|}
\hline $\mathrm{C}$ & -10.39762200 & -0.49971300 & -0.18382300 \\
\hline $\mathrm{C}$ & -8.91244900 & -0.41325500 & -0.07393500 \\
\hline $\mathrm{O}$ & -11.08718700 & 0.36890300 & -0.65891500 \\
\hline $\mathrm{O}$ & -10.86581200 & -1.64907400 & 0.30532800 \\
\hline $\mathrm{C}$ & -8.20824700 & -1.51567800 & 0.50806200 \\
\hline $\mathrm{N}$ & -7.62889000 & -2.40335600 & 0.97817500 \\
\hline $\mathrm{C}$ & -8.30280300 & 0.70867800 & -0.53120100 \\
\hline $\mathrm{C}$ & -6.91150500 & 1.06226900 & -0.55457000 \\
\hline S & -5.60085900 & 0.07580000 & 0.02181000 \\
\hline $\mathrm{C}$ & -6.44089200 & 2.26542900 & -1.06025600 \\
\hline $\mathrm{C}$ & -5.04339100 & 2.39125000 & -0.98592900 \\
\hline $\mathrm{C}$ & -4.43392900 & 1.28319000 & -0.41776200 \\
\hline $\mathrm{C}$ & -3.01074000 & 1.07473700 & -0.18565500 \\
\hline $\mathrm{C}$ & -2.06317300 & 1.93732600 & -0.78031500 \\
\hline $\mathrm{C}$ & -2.54442500 & 0.02022200 & 0.62886000 \\
\hline $\mathrm{C}$ & -0.71272900 & 1.76369000 & -0.56764400 \\
\hline $\mathrm{H}$ & -2.38947400 & 2.73514900 & -1.44520400 \\
\hline $\mathrm{C}$ & -1.19598100 & -0.17431500 & 0.84064600 \\
\hline $\mathrm{H}$ & -3.25159800 & -0.64278000 & 1.12828900 \\
\hline $\mathrm{C}$ & -0.25934400 & 0.70007500 & 0.24542100 \\
\hline $\mathrm{H}$ & 0.007 & 2.4 & 329500 \\
\hline $\mathrm{H}$ & -0.85339300 & -0.97010400 & 1.50096800 \\
\hline $\mathrm{N}$ & 1.10267000 & 0.51335500 & 0.45618700 \\
\hline $\mathrm{C}$ & 1.63123700 & -0.78545900 & 0.62034600 \\
\hline $\mathrm{C}$ & 2.64323300 & -1.00715500 & 1.56821700 \\
\hline $\mathrm{C}$ & 1.15042500 & 362100 & -0.17460300 \\
\hline $\mathrm{C}$ & 3.16969300 & 55800 & 1.72300400 \\
\hline $\mathrm{H}$ & 2.99468100 & -0.18743400 & 2.19475600 \\
\hline $\mathrm{C}$ & 1.68141900 & 1026300 & 235900 \\
\hline $\mathrm{H}$ & 0.38985400 & -1.64914500 & -0.93220500 \\
\hline $\mathrm{C}$ & 2.68758500 & -3.31777200 & 63400 \\
\hline $\mathrm{H}$ & 3.94745100 & -2.46549300 & 2.46192300 \\
\hline $\mathrm{H}$ & 1.33286300 & -3.92905600 & -0.64973600 \\
\hline $\mathrm{C}$ & 1.98037800 & 1.62052300 & 0.49585600 \\
\hline $\mathrm{C}$ & 3.24046800 & 1.51951200 & -0.11394800 \\
\hline C & 1.58951000 & 2.79870300 & 1.15204500 \\
\hline $\mathrm{C}$ & 4.10572800 & 2.60318800 & -0.07613500 \\
\hline $\mathrm{H}$ & 3.53402800 & 0.60310500 & -0.63300200 \\
\hline $\mathrm{C}$ & 2.45837700 & 3.87923000 & 1.19281900 \\
\hline $\mathrm{H}$ & 0.62224500 & 2.85354300 & 1.65173300 \\
\hline $\mathrm{C}$ & 3.70767300 & 3.77242600 & 0.57631700 \\
\hline $\mathrm{H}$ & 5.07988400 & 2.54054300 & -0.55974900 \\
\hline $\mathrm{H}$ & 2.17338900 & 4.79256000 & 1.71261500 \\
\hline $\mathrm{Br}$ & 3.43994700 & -5.04441800 & 1.10402300 \\
\hline $\mathrm{Br}$ & 4.89261300 & 5.24824700 & 0.63517300 \\
\hline $\mathrm{H}$ & -8.97817900 & 1.46150300 & -0.94761900 \\
\hline $\mathrm{H}$ & -11.83442900 & -1.64286000 & 0.20364900 \\
\hline $\mathrm{H}$ & -7.10606700 & 3.02573500 & -1.46669100 \\
\hline $\mathrm{H}$ & -4.50131800 & 3.27299400 & -1.31987700 \\
\hline I & 5.06533800 & -1.58186800 & -1.86312800 \\
\hline
\end{tabular}

\begin{tabular}{|c|c|c|c|}
\hline $\mathrm{C}$ & -10.84405600 & 0.20356100 & 0.15514900 \\
\hline $\mathrm{C}$ & -9.35620100 & 0.15969400 & 0.05615900 \\
\hline $\mathrm{O}$ & -11.51396100 & -0.69619200 & 0.59939000 \\
\hline $\mathrm{O}$ & -11.33889400 & 1.35355200 & -0.30529200 \\
\hline $\mathrm{C}$ & -8.67706800 & 1.29590400 & -0.48953500 \\
\hline $\mathrm{N}$ & -8.11787600 & 2.21107400 & -0.93043300 \\
\hline $\mathrm{C}$ & -8.72038200 & -0.95737500 & 0.48885800 \\
\hline $\mathrm{C}$ & -7.32028400 & -1.27405700 & 0.51630500 \\
\hline S & -6.03115800 & -0.23873300 & -0.02218000 \\
\hline $\mathrm{C}$ & -6.82228400 & -2.47656100 & 0.99684600 \\
\hline $\mathrm{C}$ & -5.42129500 & -2.56319100 & 0.93381300 \\
\hline $\mathrm{C}$ & -4.83662100 & -1.42557000 & 0.39894400 \\
\hline $\mathrm{C}$ & -3.41752000 & -1.17384600 & 0.18708500 \\
\hline $\mathrm{C}$ & -2.97117500 & -0.09118200 & -0.60136100 \\
\hline $\mathrm{C}$ & -2.45334800 & -2.02167200 & 0.77644000 \\
\hline $\mathrm{C}$ & -1.62631800 & 0.14528300 & -0.79157400 \\
\hline $\mathrm{H}$ & -3.69079100 & 0.56096400 & -1.09727400 \\
\hline $\mathrm{C}$ & -1.10594200 & -1.80583100 & 0.58532500 \\
\hline $\mathrm{H}$ & -2.76555900 & -2.84106600 & 1.42156200 \\
\hline $\mathrm{C}$ & -0.67239600 & -0.71360600 & -0.20067300 \\
\hline $\mathrm{H}$ & -1.29821300 & 0.96316800 & -1.43195900 \\
\hline $\mathrm{H}$ & -0.37372900 & -2.44420600 & 1.07841500 \\
\hline $\mathrm{N}$ & 0.68580600 & -0.48316700 & -0.38791200 \\
\hline $\mathrm{C}$ & 1.59798500 & -1.56252000 & -0.42774800 \\
\hline $\mathrm{C}$ & 2.84247200 & -1.43272300 & 0.20720000 \\
\hline $\mathrm{C}$ & 1.25582900 & -2.74235100 & -1.10699400 \\
\hline $\mathrm{C}$ & 3.73935800 & -2.49104800 & 0.17273400 \\
\hline $\mathrm{H}$ & 3.09970200 & -0.51536200 & 0.74351700 \\
\hline $\mathrm{C}$ & 2.15734300 & -3.79687500 & -1.14341900 \\
\hline $\mathrm{H}$ & 0.30056800 & -2.81899700 & -1.62677500 \\
\hline $\mathrm{C}$ & 3.39256300 & -3.66646800 & -0.50048400 \\
\hline $\mathrm{H}$ & 4.69970400 & -2.39856700 & 0.67883200 \\
\hline $\mathrm{H}$ & 1.90139400 & -4.70819000 & -1.68189600 \\
\hline $\mathrm{C}$ & 1.17908700 & 0.83346400 & -0.52148200 \\
\hline $\mathrm{C}$ & 2.19863200 & 1.10043300 & -1.44862000 \\
\hline $\mathrm{C}$ & 0.65799600 & 1.85790400 & 0.28474700 \\
\hline $\mathrm{C}$ & 2.69330900 & 2.38979200 & -1.56995700 \\
\hline $\mathrm{H}$ & 2.58205900 & 0.30265500 & -2.08483700 \\
\hline $\mathrm{C}$ & 1.15842900 & 3.14609000 & 0.16507000 \\
\hline $\mathrm{H}$ & -0.10904200 & 1.63426200 & 1.02646200 \\
\hline $\mathrm{C}$ & 2.17491300 & 3.40330000 & -0.75835900 \\
\hline $\mathrm{H}$ & 3.47748900 & 2.60223100 & -2.29500100 \\
\hline $\mathrm{H}$ & 0.77236700 & 3.93758400 & 0.80564700 \\
\hline I & 4.75355400 & -5.26285200 & -0.54978300 \\
\hline I & 2.96799600 & 5.33805500 & -0.90225100 \\
\hline $\mathrm{H}$ & -9.37855200 & -1.73917700 & 0.87858500 \\
\hline $\mathrm{H}$ & -12.30774000 & 1.31867500 & -0.21210800 \\
\hline $\mathrm{H}$ & -7.47056900 & -3.26424900 & 1.37770400 \\
\hline $\mathrm{H}$ & -4.85873300 & -3.43805800 & 1.25164300 \\
\hline & 4.57934400 & 1.68461800 & 2.02205700 \\
\hline
\end{tabular}


Dye- $\mathbf{F}^{+} \ldots \mathrm{I}^{-}$Interaction at amine

$\mathrm{E}(\mathrm{UM} 062 \mathrm{X})=-2153.8264374600$ a.u.

BSSE Energy $=0.0002388574 a . u$.

BSSE Corrected E(UM062X) $=-2153.8261986026$ a.u.
Dye- $\mathrm{Cl}^{+} \ldots \mathrm{I}^{-}$Interaction at amine

$\mathrm{E}(\mathrm{UM} 062 \mathrm{X})=-2874.5425463200 a . u$. BSSE Energy $=0.0002701864$ a.u.

BSSE Corrected E(UM062X) $=-2874.5422761336$ a.u.

\begin{tabular}{|c|c|c|c|}
\hline $\mathrm{C}$ & -8.99729700 & 0.37839300 & -0.03299700 \\
\hline $\mathrm{C}$ & -7.51487500 & 0.30945400 & -0.14174500 \\
\hline $\mathrm{O}$ & -9.54548800 & -0.80035500 & 0.27363600 \\
\hline $\mathrm{O}$ & -9.62730900 & 1.39363200 & -0.20440200 \\
\hline C & -6.88453700 & 1.55601400 & -0.46350100 \\
\hline $\mathrm{N}$ & -6.36907300 & 2.56122000 & -0.72321600 \\
\hline $\mathrm{C}$ & -6.83055300 & -0.84693800 & 0.05060000 \\
\hline $\mathrm{C}$ & -5.41755700 & -1.10003400 & -0.01201800 \\
\hline S & -4.18267400 & 0.07631700 & -0.35299100 \\
\hline $\mathrm{C}$ & -4.86096900 & -2.35204400 & 0.20473300 \\
\hline $\mathrm{C}$ & -3.45963900 & -2.36489900 & 0.09773000 \\
\hline $\mathrm{C}$ & -2.93299000 & -1.11927100 & -0.20520600 \\
\hline $\mathrm{C}$ & -1.53038100 & -0.76887200 & -0.39150600 \\
\hline $\mathrm{C}$ & -1.15040600 & 0.47448300 & -0.94191600 \\
\hline $\mathrm{C}$ & -0.51774800 & -1.68092900 & -0.02208800 \\
\hline $\mathrm{C}$ & 0.17780500 & 0.80067200 & -1.11563800 \\
\hline $\mathrm{H}$ & -1.90952700 & 1.18634700 & -1.26765100 \\
\hline $\mathrm{C}$ & 0.81435600 & -1.37341400 & -0.19766800 \\
\hline $\mathrm{H}$ & -0.77841900 & -2.63053100 & 0.44226000 \\
\hline $\mathrm{C}$ & 1.18199000 & -0.12328700 & -0.74771700 \\
\hline $\mathrm{H}$ & 0.45326000 & 1.74778800 & -1.57790900 \\
\hline $\mathrm{H}$ & 1.58777500 & -2.06255300 & 0.13793400 \\
\hline $\mathrm{N}$ & 2.52141200 & 0.19425400 & -0.92540000 \\
\hline $\mathrm{C}$ & 3.46862500 & -0.81805600 & -1.19377200 \\
\hline $\mathrm{C}$ & 4.72493700 & -0.77317500 & -0.56551700 \\
\hline $\mathrm{C}$ & 3.15059000 & -1.84919900 & -2.09332200 \\
\hline $\mathrm{C}$ & 5.65861600 & -1.76307800 & -0.83047600 \\
\hline $\mathrm{H}$ & 4.94152500 & 0.01660200 & 0.15317600 \\
\hline $\mathrm{C}$ & 4.08356000 & -2.83957400 & -2.35989600 \\
\hline $\mathrm{H}$ & 2.18526900 & -1.85312800 & -2.59935700 \\
\hline $\mathrm{C}$ & 5.31692100 & -2.77527100 & -1.72085100 \\
\hline $\mathrm{H}$ & 6.63524300 & -1.76850800 & -0.34935500 \\
\hline $\mathrm{H}$ & 3.87576100 & -3.64475400 & -3.06241400 \\
\hline $\mathrm{C}$ & 2.96288700 & 1.53054300 & -0.81110100 \\
\hline $\mathrm{C}$ & 3.93374300 & 2.01744900 & -1.70244300 \\
\hline $\mathrm{C}$ & 2.43655300 & 2.35304800 & 0.19975800 \\
\hline $\mathrm{C}$ & 4.37229200 & 3.32747000 & -1.58987400 \\
\hline $\mathrm{H}$ & 4.31906100 & 1.37395900 & -2.49314100 \\
\hline $\mathrm{C}$ & 2.87546400 & 3.66349700 & 0.31421800 \\
\hline $\mathrm{H}$ & 1.72155300 & 1.94223000 & 0.91212400 \\
\hline $\mathrm{C}$ & 3.83288400 & 4.12203500 & -0.58327600 \\
\hline $\mathrm{H}$ & 5.11153900 & 3.74217300 & -2.27296200 \\
\hline $\mathrm{H}$ & 2.50112800 & 4.32334800 & 1.09509600 \\
\hline $\mathrm{F}$ & 6.22172500 & -3.73387600 & -1.97928300 \\
\hline $\mathrm{F}$ & 4.25976400 & 5.39087100 & -0.47269900 \\
\hline $\mathrm{H}$ & -5.46808700 & -3.22750900 & 0.43024400 \\
\hline $\mathrm{H}$ & -2.85583500 & -3.26116500 & 0.22043500 \\
\hline $\mathrm{H}$ & -7.43399200 & -1.72540800 & 0.28948400 \\
\hline $\mathrm{H}$ & -10.50844700 & -0.66899400 & 0.32593900 \\
\hline & 2.92758400 & -0.40695400 & 2.81983400 \\
\hline
\end{tabular}

\begin{tabular}{|c|c|c|c|}
\hline $\mathrm{C}$ & -9.25826100 & 0.14083500 & -0.14024600 \\
\hline $\mathrm{C}$ & -7.77081500 & 0.18588000 & -0.24706600 \\
\hline $\mathrm{O}$ & -9.88321300 & -0.86132200 & 0.10597300 \\
\hline $\mathrm{O}$ & -9.80739100 & 1.33847400 & -0.34845200 \\
\hline $\mathrm{C}$ & -7.14892700 & 1.44004200 & -0.54745100 \\
\hline $\mathrm{N}$ & -6.63648800 & 2.45146500 & -0.79040300 \\
\hline $\mathrm{C}$ & -7.08115500 & -0.96541900 & -0.05108900 \\
\hline $\mathrm{C}$ & -5.66758800 & -1.21410200 & -0.08783900 \\
\hline S & -4.43498800 & -0.03166300 & -0.41020200 \\
\hline $\mathrm{C}$ & -5.10875200 & -2.46346600 & 0.14471600 \\
\hline $\mathrm{C}$ & -3.70698600 & -2.46840300 & 0.06663500 \\
\hline $\mathrm{C}$ & -3.18067000 & -1.21948600 & -0.23230800 \\
\hline $\mathrm{C}$ & -1.78016600 & -0.86358700 & -0.39011400 \\
\hline $\mathrm{C}$ & -1.39394400 & 0.38606300 & -0.92840000 \\
\hline $\mathrm{C}$ & -0.76859700 & -1.77278300 & -0.00401800 \\
\hline $\mathrm{C}$ & -0.06685700 & 0.72017700 & -1.07243100 \\
\hline $\mathrm{H}$ & -2.15012800 & 1.09383700 & -1.26931200 \\
\hline $\mathrm{C}$ & 0.56303700 & -1.45614300 & -0.14524900 \\
\hline $\mathrm{H}$ & -1.03364600 & -2.72562200 & 0.45086600 \\
\hline $\mathrm{C}$ & 0.93955900 & -0.19850800 & -0.68262100 \\
\hline $\mathrm{H}$ & 0.21065200 & 1.66897300 & -1.52935200 \\
\hline $\mathrm{H}$ & 1.33033000 & -2.14158900 & 0.21011700 \\
\hline $\mathrm{N}$ & 2.27291100 & 0.12764400 & -0.82308000 \\
\hline $\mathrm{C}$ & 3.25145300 & -0.88237400 & -0.99338100 \\
\hline $\mathrm{C}$ & 4.45505900 & -0.80014900 & -0.27822200 \\
\hline $\mathrm{C}$ & 3.01538100 & -1.93979100 & -1.88321200 \\
\hline $\mathrm{C}$ & 5.41761700 & -1.78544000 & -0.44323700 \\
\hline $\mathrm{H}$ & 4.61189500 & 0.01597700 & 0.42670200 \\
\hline $\mathrm{C}$ & 3.97918100 & -2.92446800 & -2.05025800 \\
\hline $\mathrm{H}$ & 2.09027600 & -1.97395000 & -2.45891600 \\
\hline $\mathrm{C}$ & 5.16879000 & -2.83770400 & -1.32610900 \\
\hline $\mathrm{H}$ & 6.35106400 & -1.74721700 & 0.11590200 \\
\hline $\mathrm{H}$ & 3.81756900 & -3.74583700 & -2.74629700 \\
\hline $\mathrm{C}$ & 2.70336500 & 1.47524600 & -0.75890600 \\
\hline $\mathrm{C}$ & 3.68998400 & 1.92555800 & -1.64850500 \\
\hline $\mathrm{C}$ & 2.15609600 & 2.33763000 & 0.20271600 \\
\hline $\mathrm{C}$ & 4.12072800 & 3.24236300 & -1.58566100 \\
\hline $\mathrm{H}$ & 4.09891700 & 1.24930900 & -2.39917200 \\
\hline $\mathrm{C}$ & 2.58920600 & 3.65483700 & 0.26685600 \\
\hline $\mathrm{H}$ & 1.42743500 & 1.95816600 & 0.91904600 \\
\hline $\mathrm{C}$ & 3.56500700 & 4.09352600 & -0.62828800 \\
\hline $\mathrm{H}$ & 4.87482000 & 3.61270500 & -2.27812700 \\
\hline $\mathrm{H}$ & 2.18552200 & 4.33400700 & 1.01605200 \\
\hline $\mathrm{Cl}$ & 6.37705300 & -4.07182700 & -1.53215600 \\
\hline $\mathrm{Cl}$ & 4.10891200 & 5.74332700 & -0.54705000 \\
\hline $\mathrm{H}$ & -7.69923800 & -1.83992300 & 0.17142200 \\
\hline $\mathrm{H}$ & -10.77282000 & 1.24206600 & -0.26314400 \\
\hline $\mathrm{H}$ & -5.71607300 & -3.34079800 & 0.36184000 \\
\hline $\mathrm{H}$ & -3.10040000 & -3.36022800 & 0.20644100 \\
\hline & 2.34248400 & -0.35739100 & 2.95984900 \\
\hline
\end{tabular}


Dye-Br ${ }^{+} \ldots \mathrm{I}^{-}$Interaction at amine

$\mathrm{E}(\mathrm{UM} 062 \mathrm{X})=-2788.1897874500$ a.u.

BSSE Energy $=0.0002704536$ a.u.

BSSE Corrected E(UM062X) $=-2788.1895169964$ a.u.
Dye-I ${ }^{+} \ldots \mathrm{I}^{-}$Interaction at amine

$\mathrm{E}(\mathrm{UM} 062 \mathrm{X})=-2545.3702278100$ a.u.

BSSE Energy $=0.0002667525$ a.u.

BSSE Corrected E(UM062X) $=-2545.3699610575$ a.u.

\begin{tabular}{|c|c|c|c|}
\hline $\mathrm{C}$ & -9.84225400 & -0.09233900 & -0.34456500 \\
\hline $\mathrm{C}$ & -8.35249100 & -0.02589100 & -0.39090000 \\
\hline $\mathrm{O}$ & -10.46087000 & -1.08341900 & -0.04368000 \\
\hline $\mathrm{O}$ & -10.40130600 & 1.07253400 & -0.67603700 \\
\hline $\mathrm{C}$ & -7.73889700 & 1.21390900 & -0.76006500 \\
\hline $\mathrm{N}$ & -7.23297700 & 2.21392600 & -1.05776300 \\
\hline $\mathrm{C}$ & -7.65297400 & -1.14576600 & -0.08098500 \\
\hline $\mathrm{C}$ & -6.23541200 & -1.37029200 & -0.04426100 \\
\hline S & -5.00894600 & -0.19251000 & -0.40445300 \\
\hline $\mathrm{C}$ & -5.66759000 & -2.59041200 & 0.29615000 \\
\hline $\mathrm{C}$ & -4.26398900 & -2.57720500 & 0.26808200 \\
\hline $\mathrm{C}$ & -3.74481500 & -1.34361000 & -0.09907600 \\
\hline $\mathrm{C}$ & -2.34465200 & -0.97682800 & -0.23567100 \\
\hline $\mathrm{C}$ & -1.33284500 & -1.85321900 & 0.21972700 \\
\hline $\mathrm{C}$ & -1.95891100 & 0.24958000 & 479700 \\
\hline $\mathrm{C}$ & -0.00157600 & -1.52956000 & 0.09067500 \\
\hline $\mathrm{H}$ & -1.59787000 & -2.78613100 & 0.71415000 \\
\hline $\mathrm{C}$ & -0.63213200 & 0.59157 & 5505900 \\
\hline $\mathrm{H}$ & -2.71383900 & 0.9319 & $\$ 48400$ \\
\hline $\mathrm{C}$ & 0.373 & -0.297 & 40800 \\
\hline $\mathrm{H}$ & 34700 & -2.18889700 & 30900 \\
\hline $\mathrm{H}$ & -0.3 & 1.520 & 82700 \\
\hline $\mathrm{N}$ & 1.708 & 0.0315 & 737300 \\
\hline $\mathrm{C}$ & 2.12962600 & 1.38248 & 419700 \\
\hline $\mathrm{C}$ & 3.12991800 & 1.8010 & 17900 \\
\hline $\mathrm{C}$ & 1.55698800 & 2.28284 & 69900 \\
\hline $\mathrm{C}$ & 3.55000900 & 3.12320700 & 202700 \\
\hline $\mathrm{H}$ & 3.55 & 1.096 & 1200 \\
\hline $\mathrm{C}$ & 1.97906300 & 3.605 & 6100 \\
\hline $\mathrm{H}$ & 0.816 & 1.930 & 44100 \\
\hline $\mathrm{C}$ & 2.969 & 4.012 & 63400 \\
\hline $\mathrm{H}$ & 4.31 & 3.462 & 59900 \\
\hline $\mathrm{H}$ & 1.55158000 & 4.309 & 09200 \\
\hline $\mathrm{C}$ & 2.69051400 & -0.98077300 & -0.76399800 \\
\hline $\mathrm{C}$ & 3.89238400 & -0.86848400 & 012900 \\
\hline $\mathrm{C}$ & 2.45911300 & -2.07180100 & -1.61361100 \\
\hline $\mathrm{C}$ & 4.85877500 & -1.856 & 7723100 \\
\hline $\mathrm{H}$ & 4.04551100 & -0.02666600 & 495900 \\
\hline $\mathrm{C}$ & 3.42660100 & -3.05892300 & -1.74317900 \\
\hline $\mathrm{H}$ & 1.53512100 & -2.13036700 & -2.18923000 \\
\hline $\mathrm{C}$ & 4.61601900 & -2.94209900 & -1.02177900 \\
\hline $\mathrm{H}$ & 5.78921100 & -1.78898900 & 0.38442400 \\
\hline $\mathrm{H}$ & 3.26303500 & -3.90345700 & -2.41054300 \\
\hline $\mathrm{Br}$ & 3.54773600 & 5.81787200 & -0.57473300 \\
\hline $\mathrm{Br}$ & 5.93822100 & -4.28817100 & -1.19988100 \\
\hline $\mathrm{H}$ & -8.26522300 & -2.01339100 & 0.18110500 \\
\hline $\mathrm{H}$ & -11.36788600 & 0.96334300 & -0.62747200 \\
\hline $\mathrm{H}$ & -6.26994500 & -3.46043100 & 0.55278600 \\
\hline $\mathrm{H}$ & -3.65078900 & -3.44693900 & 0.49236100 \\
\hline & 1.79435200 & -0.27647900 & 3.15752100 \\
\hline
\end{tabular}

\begin{tabular}{|c|c|c|c|}
\hline $\mathrm{C}$ & -10.30751700 & -0.43089800 & -0.49158500 \\
\hline $\mathrm{C}$ & -8.81900900 & -0.32851700 & -0.50381000 \\
\hline $\mathrm{O}$ & -10.90897500 & -1.42671600 & -0.17223100 \\
\hline $\mathrm{O}$ & -10.88621600 & 0.70773000 & -0.87607700 \\
\hline $\mathrm{C}$ & -8.22679800 & 0.91312100 & -0.90090200 \\
\hline $\mathrm{N}$ & -7.73806500 & 1.91478400 & -1.22091100 \\
\hline $\mathrm{C}$ & -8.10055300 & -1.41948100 & -0.13891800 \\
\hline $\mathrm{C}$ & -6.67928200 & -1.60706400 & -0.06065600 \\
\hline S & -5.47346300 & -0.41080700 & -0.42830600 \\
\hline $\mathrm{C}$ & -6.09070700 & -2.80163300 & 0.33167400 \\
\hline $\mathrm{C}$ & -4.68774900 & -2.75429100 & 0.33669200 \\
\hline $\mathrm{C}$ & -4.18923400 & -1.51970400 & -0.05607400 \\
\hline $\mathrm{C}$ & -2.79600000 & -1.12241700 & -0.17128400 \\
\hline $\mathrm{C}$ & -2.42801400 & 0.10820500 & -0.76380300 \\
\hline $\mathrm{C}$ & -1.77175800 & -1.97181600 & 0.30775500 \\
\hline $\mathrm{C}$ & -1.10778400 & 0.47965800 & -0.87457300 \\
\hline $\mathrm{H}$ & -3.19118200 & 0.77092700 & -1.17281700 \\
\hline $\mathrm{C}$ & -0.44646300 & -1.61882100 & 0.19810500 \\
\hline $\mathrm{H}$ & -2.02292700 & -2.90779300 & 0.80353700 \\
\hline $\mathrm{C}$ & -0.08891400 & -0.38220600 & -0.39756000 \\
\hline $\mathrm{H}$ & -0.84261700 & 1.41089500 & -1.37302800 \\
\hline $\mathrm{H}$ & 0.32839400 & -2.25823600 & 0.61725200 \\
\hline $\mathrm{N}$ & 1.23875600 & -0.02448300 & -0.51479700 \\
\hline $\mathrm{C}$ & 2.24727100 & -1.01480300 & -0.61276400 \\
\hline $\mathrm{C}$ & 3.43274500 & -0.86630900 & 0.12057600 \\
\hline $\mathrm{C}$ & 2.05822100 & -2.11916200 & -1.45480300 \\
\hline $\mathrm{C}$ & 4.42518700 & -1.83215800 & 0.01986000 \\
\hline $\mathrm{H}$ & 3.55462700 & -0.01393900 & 0.78888700 \\
\hline $\mathrm{C}$ & 3.05269300 & -3.08355200 & -1.55697000 \\
\hline $\mathrm{H}$ & 1.14651800 & -2.20649300 & -2.04645500 \\
\hline $\mathrm{C}$ & 4.22860800 & -2.93437400 & -0.81710700 \\
\hline $\mathrm{H}$ & 5.34108400 & -1.72839800 & 0.59971700 \\
\hline $\mathrm{H}$ & 2.91360700 & -3.93540100 & -2.22097700 \\
\hline $\mathrm{C}$ & 1.62905700 & 1.33650200 & -0.51270900 \\
\hline $\mathrm{C}$ & 2.63094900 & 1.76735100 & -1.39418700 \\
\hline $\mathrm{C}$ & 1.02430800 & 2.23400000 & 0.37930100 \\
\hline $\mathrm{C}$ & 3.01950100 & 3.09978500 & -1.39269300 \\
\hline $\mathrm{H}$ & 3.08539900 & 1.06398300 & -2.09222300 \\
\hline $\mathrm{C}$ & 1.41623700 & 3.56668500 & 0.38134200 \\
\hline $\mathrm{H}$ & 0.28342200 & 1.87306700 & 1.09300600 \\
\hline $\mathrm{C}$ & 2.40894400 & 3.99138600 & -0.50502400 \\
\hline $\mathrm{H}$ & 3.78660800 & 3.44038500 & -2.08652700 \\
\hline $\mathrm{H}$ & 0.95865200 & 4.26293400 & 1.08273900 \\
\hline I & 5.73564200 & -4.38902500 & -0.97287900 \\
\hline I & 3.00164200 & 6.00539000 & -0.50122800 \\
\hline $\mathrm{H}$ & -8.69773500 & -2.29302800 & 0.13775600 \\
\hline $\mathrm{H}$ & -11.85078700 & 0.57585000 & -0.84791500 \\
\hline $\mathrm{H}$ & -6.67814100 & -3.67780100 & 0.60164000 \\
\hline $\mathrm{H}$ & -4.05998000 & -3.60101000 & 0.60414600 \\
\hline I & 1.23202800 & -0.29221700 & 3.28696100 \\
\hline
\end{tabular}




\section{References}

(1) Swords, W. B.; Simon, S. J. C.; Parlane, F. G. L.; Dean, R. K.; Kellett, C. W.; Hu, K.; Meyer, G. J.; Berlinguette, C. P. Evidence for Interfacial Halogen Bonding. Angewandte Chemie - International Edition 2016, 55, 5956-5960.

(2) Bonhôte, P.; Dias, A. P.; Armand, M.; Papageorgiou, N.; Kalyanasundaram, K.; Grätzel, M. Hydrophobic, Highly Conductive Ambient-Temperature Molten Salts. Inorg. Chem. 1996, 35, 1168-1178.

(3) Robson, K. C. D.; Hu, K.; Meyer, G. J.; Berlinguette, C. P. Atomic Level Resolution of Dye Regeneration in the Dye Sensitized Solar Cell. J. Am. Chem. Soc. 2013, 135, 1961-1971.

(4) Heimer, T.; Castellano, F. N.; Meyer, G. J. Enhanced Spectral Sensitivity from Ruthenium(II) Polypyridyl Based Photovoltaic Devices. Inorg. Chem. 1994, 33, 5741-5749.

(5) Gaussian 09, Revision E.01, Frisch, M. J.; Trucks, G. W.; Schlegel, H. B.; Scuseria, G. E.; Robb, M. A.; Cheeseman, J. R.; Scalmani, G.; Barone, V.; Mennucci, B.; Petersson, G. A.; Nakatsuji, H.; Caricato, M.; Li, X.; Hratchian, H. P.; Izmaylov, A. F.; Bloino, J.; Zheng, G.; Sonnenberg, J. L.; Hada, M.; Ehara, M.; Toyota, K.; Fukuda, R.; Hasegawa, J.; Ishida, M.; Nakajima, T.; Honda, Y.; Kitao, O.; Nakai, H.; Vreven, T.; Montgomery, J. A., Jr.; Peralta, J. E.; Ogliaro, F.; Bearpark, M.; Heyd, J. J.; Brothers, E.; Kudin, K. N.; Staroverov, V. N.; Kobayashi, R.; Normand, J.; Raghavachari, K.; Rendell, A.; Burant, J. C.; Iyengar, S. S.; Tomasi, J.; Cossi, M.; Rega, N.; Millam, J. M.; Klene, M.; Knox, J. E.; Cross, J. B.; Bakken, V.; Adamo, C.; Jaramillo, J.; Gomperts, R.; Stratmann, R. E.; Yazyev, O.; Austin, A. J.; Cammi, R.; Pomelli, C.; Ochterski, J. W.; Martin, R. L.; Morokuma, K.; Zakrzewski, V. G.; Voth, G. A.; Salvador, P.; Dannenberg, J. J.; Dapprich, S.; Daniels, A. D.; Farkas, O.; Foresman, J. B.; Ortiz, J. V.; Cioslowski, J.; Fox, D. J. Gaussian, Inc., Wallingford CT, 2009.

(6) Zhao, Y.; Truhlar, D. G. The M06 suite of density functionals for main group thermochemistry, thermochemical kinetics, noncovalent interactions, excited states, and transition elements: Two new functionals and systematic testing of four M06-class functionals and 12 other function. Theor. Chem. Acc. 2008, 120, 215-241.

(7) Zhao, Y.; Truhlar, D. G. Density functionals with broad applicability in chemistry. Acc. Chem. Res. 2008, 41, 157-167.

(8) Barone, V.; Cossi, M. Quantum calculation of molecular energies and energy gradients in solution by a conductor solvent model. J. Phys. Chem. A 1998, 102, 1995-2001.

(9) Cossi, M.; Rega, N.; Scalmani, G.; Barone, V. Energies, structures, and electronic properties of molecules in solution with the C-PCM solvation model. J. Comput. Chem. 2003, 24, 669-681. 
(10) Jmol: an open-source Java viewer for chemical structures in 3D. http://www.jmol.org/.

(11) Dennington, R.; Keith, T.; Millam, J. GaussView Version 5., Semichem Inc. Shawnee Mission KS 2009.

(12) Dunning Jr, T. H. Gaussian basis sets for use in correlated molecular calculations. I. The atoms boron through neon and hydrogen. J. Chem. Phys. 1989, 90, 1007-1023.

(13) Kendall, R. A.; Dunning Jr., T. H.; Harrison, R. J. Electron affinities of the first-row atoms revisited. Systematic basis sets and wave functions. J. Chem. Phys. 1992, 96, 6796-6806.

(14) Dunning T.H., J.; Peterson, K. A.; Wilson, A. K. Gaussian basis sets for use in correlated molecular calculations. X. The atoms aluminum through argon revisited. J. Chem. Phys. 1993, 98, 1358-15.

(15) Peterson, K. A.; Woon, D. E.; Dunning, T. H. Benchmark calculations with correlated molecular wave functions. IV. The classical barrier height of the $\mathrm{H}+\mathrm{H} 2 \mathrm{H} 2+\mathrm{H}$ reaction. The J. Chem. Phys. 1994, $100,7410-7415$.

(16) Hashimoto, T.; Hirao, K.; Tatewaki, H. Comment on Dunning's correlation-consistent basis sets. Chem. Phys. Lett. 1995, 243, 190-192.

(17) Peterson, K. A. Systematically convergent basis sets with relativistic pseudopotentials. I. Correlation consistent basis sets for the post-d group 13-15 elements. J. Chem. Phys. 2003, 119, 11099-11112.

(18) Peterson, K. A.; Shepler, B. C.; Figgen, D.; Stoll, H. On the spectroscopic and thermochemical properties of $\mathrm{ClO}, \mathrm{BrO}, \mathrm{IO}$, and their anions. J. Phys. Chem. A 2006, 110, 13877-13883.

(19) Feller, D. The role of databases in support of computational chemistry calculations. J. Comput. Chem. 1996, 17, 1571-1586.

(20) Schuchardt, K. L.; Didier, B. T.; Elsethagen, T.; Sun, L.; Gurumoorthi, V.; Chase, J.; Li, J.; Windus, T. L. Basis set exchange: A community database for computational sciences. J. Chem. Inf. Model. 2007, 47, 1045-1052.

(21) Boys, S.; Bernardi, F The calculation of small molecular interactions by the differences of separate total energies. Some procedures with reduced errors. Mol. Phys. 1970, 19, 553-566.

(22) Simon, S.; Duran, M.; Dannenberg, J. J. How does basis set superposition error change the potential surfaces for hydrogen-bonded dimers? The J. Chem. Phys. 1996, 105, 11024-11031.

(23) Lipparini, F.; Scalmani, G.; Mennucci, B. Non covalent interactions in RNA and DNA base pairs: a quantum-mechanical study of the coupling between solvent and electronic density. Phys. Chem. Chem. Phys. 2009, 11, 11617-11623. 
(24) Zawada, A.; Góra, R. W.; Mikołajczyk, M. M.; Bartkowiak, W. On the Calculations of Interaction Energies and Induced Electric Properties within the Polarizable Continuum Model. J. Phys. Chem. A 2012, 116, 4409-4416.

(25) NBO Version 6.0. Glendening, E. D.; Badenhoop, J. K.; Reed, A. E.; Carpenter, J. E.; Bohmann, J. A.; Morales, C. M.; Landis, C. R.; Weinhold, F. Theoretical Chemistry Institute, University of Wisconsin, Madison (2013).

(26) Glendening, E. D.; Landis, C. R.; Weinhold, F. NBO 6.0: Natural bond orbital analysis program. J. Comput. Chem. 2013, 34, 1429-1437.

(27) Carpenter, J. E. Extension of Lewis structure concepts to open-shell and excited-state molecular species, Ph. D. thesis, University of Wisconsin, Madison, WI, 1987.

(28) Weinhold, F. and Carpenter, J. E. in The Structure of Small Molecules and Ions, R. Naaman, and Z. Vager, Ed.; Plenum, 1988; 227-236.

(29) Foster, J. P.; Weinhold, F Natural Hybrid Orbitals. J. Am. Chem. Soc. 1980, 102, 7211-7218.

(30) Reed, A. E.; Weinhold, F. Natural bond orbital analysis of near-Hartree-Fock water dimer. The J. Chem. Phys. 1983, 78, 4066-4073.

(31) Reed, A. E.; Weinstock, R. B.; Weinhold, F. Natural population analysis. J. Chem. Phys. 1985, 83, $735-746$.

(32) Reed, A. E.; Weinhold, F Natural localized molecular orbitals. The J. Chem. Phys. 1985, 83, 17361740 .

(33) Reed, A. E.; Curtiss, L. A.; Weinhold, F. Intermolecular interactions from a natural bond orbital, donor-acceptor viewpoint. Chem. Rev. (Washington, DC, United States) 1988, 88, 899-926.

(34) Carpenter, J. E.; Weinhold, F. Analysis of the geometry of the hydroxymethyl radical by the "different hybrids for different spins" natural bond orbital procedure. J. Mol. Struct. THEOCHEM 1988, 169, $41-62$. 